\title{
A new interpretation of the absorption and the dual fluorescence of Prodan in solution
}

\author{
Cíntia C. Vequi-Suplicy ${ }^{1,2}$, Yoelvis Orozco-Gonzalez ${ }^{1,3}$, M. Teresa Lamy ${ }^{1}$, \\ Sylvio Canuto ${ }^{1}$, Kaline Coutinho ${ }^{1 *}$ \\ 1. Instituto de Física, Universidade de São Paulo, Rua do Matão,1371, 05508-090, São Paulo, SP, \\ Brazil. \\ 2. Fundacion IMDEA-Nanociencia Cantoblanco, 28049, Madrid, Spain. \\ 3. Department of Chemistry, Georgia State University, Atlanta, GA 30302, United States.
}

\begin{abstract}
Remarkable interest is associated to the interpretation of Prodan fluorescent spectrum. A sequential hybrid Quantum Mechanics/Molecular Mechanics (S-QM/MM) method was used to establish that the fluorescent emission occurs from two different excited states, resulting in a broad asymmetric emission spectrum. The absorption spectra in several solvents were measured and calculated using different theoretical models presenting excellent agreement. All theoretical models (INDO-CIS, TDB3LYP and CASPT2) agree that the first observed band at the absorption spectrum in solution is composed of three electronic excitations very close in energy. Then, the electronic excitation around 340-360 nm may populate the first three excited states $\left(\pi-\pi^{*} L_{b}, n-\pi^{*}\right.$ and $\left.\pi-\pi^{*} L_{a}\right)$. The ground state $S_{0}$ and the first three excited states were analyzed using multi-configurational calculations (CASSCF) and second-order multi-configurational perturbation theory (CASPT2). The corresponding equilibrium geometries are all planar in vacuum. Considering the solvent effects in the electronic structure of the solute and in the solvent relaxation around the solute, it was identified that these three excited states can change the relative order depending on the solvent polarity and following the minimum path energy, internal conversions may occur. A consistent explanation of the experimental data is obtained with the conclusive interpretation that the two bands observed in the fluorescent spectrum of Prodan, in several solvents, are due to the emission from two independent states. Our results indicate that these are the $n-\pi^{*} S_{2}$ state with small dipole moment at lower emission energy and the $\pi-\pi * L_{b} S_{1}$ with large dipole moment at higher emission energy.
\end{abstract}

Keywords: Prodan, Photophysics, UV-Vis absorption, fluorescence, two state fluorescence, QM/MM.

* To whom correspondence should be addressed:

E-mail: kaline@if.usp.br

Instituto de Física, Universidade de São Paulo, Rua do Matão 1371, Cidade Universitária, 05508-090, São Paulo, SP, Brazil.

Tel: +55 1130916745 


\section{Introduction}

Prodan (2-dimethylamino-6-propionylnaphthalene, Fig. 1) and its derivatives, such as Laurdan, are widely used in biologically relevant systems ${ }^{1-7}$ as fluorescent probes. It is very sensitive to the environment with its remarkable emission spectrum shifting by about $120 \mathrm{~nm}$ $\left(6.4 \times 10^{3} \mathrm{~cm}^{-1}=0.79 \mathrm{eV}\right)$ from cyclohexane $\left(\lambda_{\max }=400 \mathrm{~nm}=25.5 \times 10^{3} \mathrm{~cm}^{-1}=3.16 \mathrm{eV}\right)$ to water $\left(\lambda_{\max }=520 \mathrm{~nm}=19.1 \times 10^{3} \mathrm{~cm}^{-1}=2.37 \mathrm{eV}\right) .{ }^{1,8-10}$ Inserted in biological membranes its emission spectra depend on the lipid bilayer phase (gel or fluid), with the wavelength of the maximum of the spectrum shifting by $50 \mathrm{~nm}\left(2.4 \times 10^{3} \mathrm{~cm}^{-1}=0.30 \mathrm{eV}\right)$ from one phase to the other. ${ }^{3,7,11}$

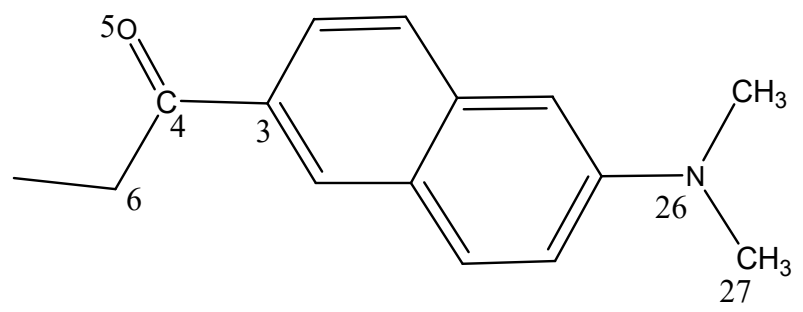

Figure 1: Prodan molecular structure.

The emission spectrum of Prodan is very peculiar because it is broad and asymmetric and is composed by dual emission in several distinct environments, from nonpolar to polar solutions $^{12-15}$ and also in biological systems. ${ }^{7,11,16}$ The explanation for these dual emission is still a matter of discussion. ${ }^{17-20}$ The common hypothesis is that the dual fluorescence comes from only one electronic state, the first excited state $S_{1}$, but with a higher emission energy coming from a solvent-non-relaxed $\mathrm{S}_{1}$ state, so-called locally excited (LE) state, and another with a lower emission energy coming from a solvent-relaxed $\mathrm{S}_{1}$ state, so-called internal charge transfer (ICT) state. Possibly also with an internal twist of the fluorophore (TICT). ${ }^{21-}$ 37 Other studies recognize some limitations of this hypothesis, suggesting that more investigation is necessary to fully understand the dual fluorescence of Prodan. ${ }^{8,10,14-19,36,38}$ Therefore, understanding the origin of the dual emission decay mechanism is critical to improve the applications of Prodan and its derivative as fluorescent probes in biological environments.

It is important to note two consequences of this common hypothesis of the dual emission of Prodan due to the solvent-non-relaxed $S_{1}$ state (higher emission energy $E_{1}$ or $\lambda_{1}{ }^{-1}$, locally excited state) and the solvent-relaxed $S_{1}$ state (lower emission energy $E_{2}$ or $\lambda_{2}{ }^{-1}$, 
charge transfer excited state): (i) as the difference between the two emitting states come from the solute-solvent relaxation during the emission process, one should expect a temperature dependence of the emission spectrum shape due to the different kinetic energy, which induces a faster relaxation and consequently an increase in the fraction of the lower energy emission de-excitation with the increase of temperature (increasing temperature $\rightarrow$ increasing $\mathrm{E}_{2}{ }^{\exp }$ intensity or area); and (ii) as the relaxed $\mathrm{S}_{1}$ state is considered to be a charge transfer excited state, one should expect a larger dipole moment that induces a better stabilization in polar solvents and consequently an increase in the fraction of the lower energy emission deexcitation with the increase of solvent polarity (increasing polarity $\rightarrow$ increasing $\mathrm{E}_{2}{ }^{\text {exp }}$ intensity or area). None of these dependencies were observed experimentally. Indeed, the behavior of the dual emission of Prodan (and its derivative Laurdan) were analyzed with respect to the temperature and the solvent polarity variations in an experimental study. ${ }^{10}$ The temperature was changed from 5 to $40^{\circ} \mathrm{C}$ and no difference was observed in either the emission spectra of Prodan or Laurdan (experimental observation: increasing temperature $\rightarrow$ no change in $\mathrm{E}_{1}{ }^{\mathrm{exp}}$ and $\mathrm{E}_{2}{ }^{\mathrm{exp}}$ ). This experimental information contradicts the first consequence of the common hypothesis discussed above. Additionally, the effect of solvent polarity was analyzed with two different and independent technics used to decompose the emission spectra: the decomposition into two Gaussian bands and the Decay Associated Spectra methodology using time resolved fluorescence. The maxima of the higher energy emission band of Prodan in several solvents were measured, $\mathrm{E}_{1}{ }^{\exp }=25.5,23.1,22.9,21.4,20.1$ and $19.1 \times 10^{3} \mathrm{~cm}^{-1}\left(\lambda_{1}{ }^{\exp }=393,433,437,467,496\right.$ and $\left.522 \mathrm{~nm}\right)$ for cyclohexane, chloroform, dichloromethane, acetonitrile, methanol and water, respectively. And for the lower energy emission band in the same solvents were measured, $\mathrm{E}_{2}{ }^{\exp }=24.1,21.8,21.5,20.3,18.9$ and $17.3 \times 10^{3} \mathrm{~cm}^{-1}\left(\lambda_{2}{ }^{\exp }=415,459,466,493,528\right.$ and $\left.579 \mathrm{~nm}\right)$, respectively. Both emission bands are red shifted by increasing the polarity of the solvent from cyclohexane to water (experimental observation: increasing polarity $\Delta \mathrm{E}_{1}$ and $\Delta \mathrm{E}_{2}$ are red shifted). These information can be seen in Fig. 2 where the experimental fluorescent emission spectra of Prodan in cyclohexane and water solutions, obtained in previous work ${ }^{10}$, are shown together with the two decomposed band for each solvent. Comparing the maximum intensity of these two decomposed bands, $\mathrm{E}_{1}{ }^{\exp }$ and $\mathrm{E}_{2}{ }^{\mathrm{exp}}$, and the band areas, it was obtained that the fraction of the lower energy band is higher in cyclohexane than in water, decreasing as the polarity of the solution increases i.e., the intensities are $0.62,0.54,0.46,0.34,0.24$ and 0.15 for the solvents respectively, and the areas fraction are $\mathrm{A}_{2} / \mathrm{A}_{\mathrm{T}}=61 \%, 52 \%, 43 \%, 36 \%, 26 \%$ and 
$14 \%$, respectively. ${ }^{10}$ Therefore, the lower energy $\mathrm{E}_{2}{ }^{\exp }$ emission is disfavored as the solvent polarity increases, i.e. the amount of photons emitted from the lower energy excited state is smaller in water than in cyclohexane. This finding is in agreement with the previous study performed in ethanol/water mixture ${ }^{14}$ and ethanol/buffer mixture. ${ }^{15}$ However, it is in conflict with the second consequence of the common hypothesis discussed above. Therefore, a new hypothesis for the dual fluorescence of Prodan (and Laurdan) in homogeneous solvents is possible, where the two emission bands would come from two different excited electronic states, ${ }^{10,14,15}$ where the lower energy $E_{2}$ emission state should have a small dipole moment (because this state is unfavored with increasing polarity) and the higher energy $\mathrm{E}_{1}$ emission state should have a large dipole moment (because this state is favored with increasing polarity). To verify this possibility, or suggest a new one, Monte Carlo and Molecular Dynamics simulations and quantum mechanics calculations were performed in this work, along with experimental measures of the Prodan spectrum in different solvents. The lowlying excited electronic states and deactivation mechanisms of Prodan were characterized in vacuum and in solvents and also the absorption and emission spectra were computed.

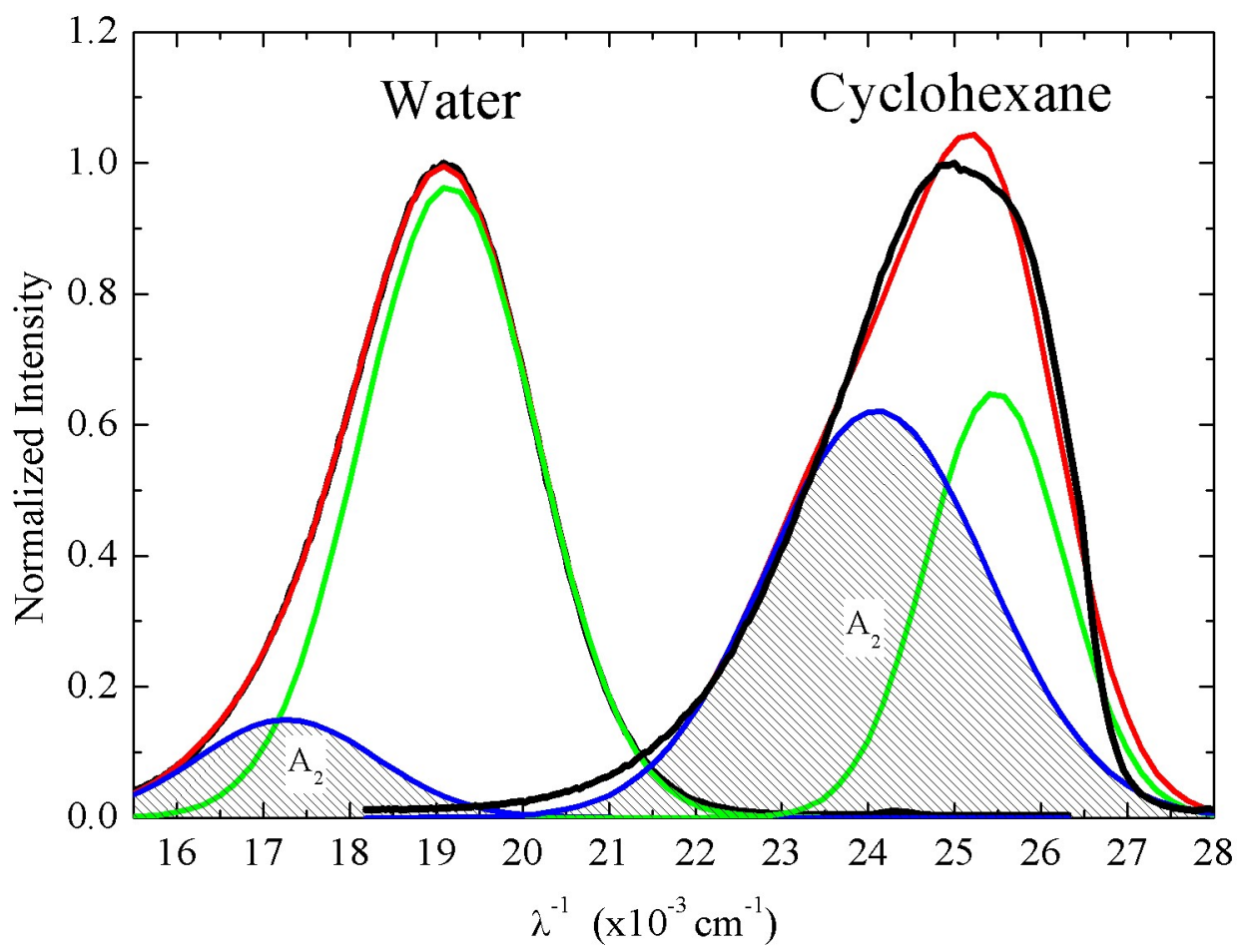

Figure 2: Experimental fluorescent emission spectra of Prodan in cyclohexane and water solutions (in black), Gaussian decomposition: higher energy $E_{1}$ or $\lambda_{1}{ }^{-1}$ band (in green), lower energy $E_{2}$ or $\lambda_{2}{ }^{-1}$ band (in blue) and total band (in red). Data obtained from ref. 10. 
Initially, the electronic transition energies of Prodan in some solvents were calculated and compared with the experimental data of the first band of the UV-visible absorption spectra. This motivated us to obtain again the experimental absorption spectrum and analyze also the broadening of the band. An excellent agreement was found between the theoretical results and the experimental data and the important conclusion obtained from this comparison was the existence of three electronic excitations in the first absorption band. Therefore, by exciting Prodan at the wavelength of the maximum absorption of the first band, our calculations show that it is possible to populate three different excited states. These combined theoretical and experimental results along with the characterization of the low-lying excited electronic states of the Prodan will be used to support the hypothesis that Prodan fluorescent emission is due to the decay of two independent states that are accessible in the absorption band and can be populated when Prodan is excited with energies around 27.8-29.5 x $10^{3} \mathrm{~cm}^{-1}$ (360-340 nm). These two states are characterized with quantum mechanics (QM) calculations using different theoretical levels ranging from semi-empirical to multi-configurational perturbation theory.

\section{Experiment}

To assist and complement our theoretical results for the absorption spectra of Prodan in different solvents these spectra were measured experimentally in this work.

\section{Materials and methods}

The fluorophores Prodan and Laurdan were purchased from Molecular Probes Inc. (Eugene, OR, USA) and the solvents cyclohexane, chloroform, dichloromethane, acetonitrile and methanol from Sigma-Aldrich (St Louis, MO, USA). Water was Milli-Q Plus (Millipore), $\mathrm{pH} \sim 6.0$. Stock solutions of the fluorophore in chloroform $(1.5 \mathrm{mM}$ ) were used in all experiments. Appropriated amounts of these solutions were transferred to glass flasks using calibrated glass micro-syringes. Chloroform was evaporated under a stream of dry $\mathrm{N}_{2}$. The dry residue was dissolved in the desired solvent to obtain the fluorophore concentration of $4.0 \mu \mathrm{M} . \mathrm{pH}$ was measured for all samples in water, and no change was observed $(\mathrm{pH} \sim$ $6.0)$.

The electronic absorption spectra were measured with a Cary-50 spectrophotometer (Varian Australia PTY LTD, Mulgrave, VIC, Australia) with temperature fixed at $25 \pm 1^{\circ} \mathrm{C}$ with a Single Cell Peltier temperature controller. All data shown are averages of at least three experiments. 


\section{Computational Details}

\section{Solute polarization in solution}

The solvent effects in the electronic states (ground and excited states) and in the transition energies were taken into account using the sequential Quantum Mechanics/Molecular Mechanics (S-QM/MM) methodology, ${ }^{39-41}$ unless polarizable continuum model (PCM) ${ }^{42}$ were stated.

The S-QM/MM methodology is a two steps procedure: first MM simulations were performed considering the solute surrounded by the solvent in a specific thermodynamic condition; then, QM calculations were performed in solute-solvent configurations obtained from the MM simulations to provide averaged electronic properties. Usually, after the simulations a statistical analysis is performed ${ }^{39}$ and 100 statistically uncorrelated solutesolvent configurations (less than $10 \%$ of statistical correlation) were selected and submitted to QM calculations to obtain averaged solute electronic properties at a specific thermodynamic condition. This procedure was successfully used for several properties such as solute polatization ${ }^{39,43}$, UV-vis absorption spectrum ${ }^{40,41,44,45}$, NMR properties ${ }^{46}$ and others, where the solvent was used in the QM calculations either as electrostatic embedding only or in addition to some closer explicit solvent molecules. For the cases where the solvent effect is treated only by the electrostatic embedding, we have shown that the average of 100 QM calculations can be represented by one QM calculation performed with the Average Solvent Electrostatic Configuration (ASEC). ${ }^{47}$ This makes it possible to compute properties of the excited electronic states in a very efficient way considering the effect of the environment.

The solute polarization was obtained using the ASEC in an iterative-QM/MM polarization procedure. ${ }^{44-47}$ The iteration starts with a MM simulation of the solute-solvent system where the classical electrostatic potential uses atomic charges of the solute obtained from the QM calculation in vacuum or from a previous PCM calculation. Then, after the simulations the solvent distribution around the solute is obtained to generate the ASEC and new QM calculation is performed recalculating new solute atomic charges to start a following iteration step. This procedure is repeated several times until reaches converged atomic charges or dipole moment. In the case of Prodan, we obtained the atomic charges with the CHELPG procedure ${ }^{48}$ for the electrostatic fitting calculated at MP2/aug-cc-pVDZ level for the ground state and with the ElectroStatic Potential Fitted method (ESPF) ${ }^{49}$ at the CASSCF/ANO-L level for the ground and excited states. Then, for both QM levels (MP2 and CASSCF), an ASEC electrostatic embedding of the solvent was used to include the electronic 
polarization of the solute ground state $\left(\mathrm{S}_{0}\right)$ in different solvents. And also, the iterative polarization procedure was performed for the first three excited electronic state $\left(\pi-\pi^{*} L_{\mathrm{a}}, \pi\right.$ $\pi^{*} \mathrm{~L}_{\mathrm{b}}$ and $\left.n-\pi^{*}\right)$ only with CASSCF to obtain the Prodan electron density in electrostatic equilibrium with the water solution, as performed before. ${ }^{44-47,50}$ It is important to note that this polarization procedure allows the relaxation of the electronic density of the solute in the presence of the solvent. Therefore, when the polarizations of the excited states are performed at the $\mathrm{S}_{0}$ geometry, the instantaneous relaxed electronic state in solution is obtained to describe the vertical excitation. But when the polarizations are performed at the corresponding equilibrium excited state geometry, the fully relaxed electronic state in solution is obtained to describe the emission transition. Here, as the excited states geometries of Prodan were optimized in vacuum, the solvent effect in the geometry relaxation was not taken into account. But to consider this geometry relaxation solvent effect, it is necessary to analyze the possibility of new force field parameters to avoid the bias and properly describe the equilibrium excited state geometries in the MM simulations.

\section{Absorption and emission spectra}

The absorption spectra were calculated using four different methods: (1) Time Dependent Density Functional Theory (TD-DFT) ${ }^{51}$ using the B3LYP functional and 6$311 \mathrm{G}(\mathrm{d})$ basis set with the solvent treated as polarizable continuum model (PCM). ${ }^{42}$ This method will be called TD-B3LYP/PCM; (2) Semi-empirical Intermediate Neglect of Differential Overlap with single excitations in the Configuration Interaction method (INDOCIS) using the original spectroscopic parameterization ${ }^{52}$ with the solvent included as SelfConsistent Reaction Field (SCRF) ${ }^{53,54}$. This method will be called INDO-CIS/SCRF; (3) Semi-empirical INDO-CIS with the solvent included as explicit molecules using solutesolvent configurations obtained from Molecular Mechanics simulation with either methods: Molecular Dynamics (MD) or Monte Carlo (MC). This method will be called INDOCIS/Explicit. It uses several solute-solvent configurations characterizing the solution at a specific thermodynamic condition. Then, naturally it provides inhomogeneous contribution to the band broadening. The details of the simulations are given below; (4) Single-state second order multi-configurational perturbation theory (CASPT2) $)^{55}$ based on a multi-state Complete Active Space Self-Consisted Field (CASSCF) wave function. ${ }^{56}$ This method was used to compute the absorption spectrum of the Prodan, but also to characterize the low-lying excited electronic states and compute the emission spectrum, both in vacuum and in water solution 
treated with PCM and with ASEC electrostatic embedding. This method will be called CASPT2/PCM and CASPT2/ASEC. The active space of CASSCF calculations correlates 12 electrons in 12 orbitals, termed as $\operatorname{CASSCF}(12,12)$. The active orbitals are the five $\pi$ and six $\pi^{*}$ that better describe the two lowest $\pi-\pi^{*}$ excited states and it was added the oxygen lone-

pair orbital to describe the lowest $n-\pi^{*}$ excited state. The ANO-L basis set ${ }^{57,58}$ was used with the contraction scheme $\mathrm{C}, \mathrm{O}$ and $\mathrm{N}(14 \mathrm{~s} 9 \mathrm{p} 4 \mathrm{~d}) /[4 \mathrm{~s} 3 \mathrm{p} 1 \mathrm{~d}], \mathrm{H}(8 \mathrm{~s} 4 \mathrm{p}) /[2 \mathrm{~s} 1 \mathrm{p}]$. The ground and excited electronic states were optimized at the same level, using CASSCF/ANO-L$4 \mathrm{~s} 3 \mathrm{p} 1 \mathrm{~d} / 2 \mathrm{~s} 1 \mathrm{p}$ in vacuum. The vibration frequencies were also calculated to ensure the equilibrium geometry of each state and the total energy was corrected by the zero-point energy and vibrational entropy.

The ground state equilibrium geometry of the Prodan was previously obtained by Vequi-Suplicy et $a l .{ }^{43}$ This planar geometry, generated with DFT/B3LYP/6-31G(d) level, does not show significant changes when optimized in vacuum or in solution (treated as PCM). This behavior persisted even using two additional basis sets $(6-311+G(d)$ and aug-ccpVDZ). For comparison with previous result this geometry was also used in the calculation of the absorption spectrum of Prodan in solution with three methods: TD-B3LYP/PCM, INDOCIS/SCRF and INDO-CIS/Explicit.

All the semi-empirical calculations were performed with the ZINDO program, ${ }^{59}$ the DFT calculations were performed with the Gaussian03 program package ${ }^{60}$ and the multiconfigurational calculations were performed with the MOLCAS 7.6 program package. ${ }^{61}$

\section{Molecular mechanics simulations}

The Molecular Mechanics simulations were performed using the standard Monte Carlo (MC) method with the Metropolis sampling technique ${ }^{62}$ with the solute rigid in the optimized geometry. Nevertheless, to take into account the effect of the intramolecular degree of freedom, Molecular Dynamics (MD) was also performed. The MD flexibility is expected to mostly affects the $\mathrm{C}=\mathrm{O}$ stretch in water due to the formation of hydrogen bonds and possible rotations between the aromatic ring and the other groups. The isothermal-isobaric $N P T$ ensemble at room temperature and pressure conditions (298 $\mathrm{K}$ and $1 \mathrm{~atm})$ was used in both the MC and MD simulations. One Prodan and 1000 water molecules or 500 molecules of the other solvents (acetonitrile, dichloromethane and cyclohexane) were considered in a rectangular box with periodic boundary conditions and minimum image method. The intramolecular parameters of Prodan used in flexible simulation were obtained from the allatom Optimized Potentials for Liquid Simulations (OPLS/AA) force field. ${ }^{63}$ However, the 
equilibrium bond distances and angles were used from the optimized geometry with QM calculation with B3LYP/6-31G(d) level and the rotational angles between the aromatic rings and the $-\mathrm{N}\left(\mathrm{CH}_{3}\right)_{2}$ and the $-\mathrm{COCH}_{2} \mathrm{CH}_{3}$ groups $\left(\varphi_{O-C-C-C}, \varphi_{C-N-C-C}\right.$ and $\left.\varphi_{H-C-N-C}\right)$ were reparametrized to describe QM energy profile with the same level. The intermolecular interactions were described by the Lennard-Jones plus Coulomb potentials with three parameters for each interacting site $\left(\varepsilon_{i}, \sigma_{i}\right.$ and $\mathrm{q}_{i}$ for an atom $\left.i\right)$. The Lennard-Jones, $\varepsilon_{i}$ and $\sigma_{i}$, parameters for the Prodan were obtained from the all-atom Optimized Potentials for Liquid Simulations (OPLS/AA) force field ${ }^{63}$ and the atomic charges, $\mathrm{q}_{i}$, of the Coulomb potential were obtained with QM calculations polarized in the presence of the solvent with an iterative$\mathrm{QM} / \mathrm{MM}$ polarization procedure ${ }^{44-47}$ as discussed in the previous section.

The geometry and the parameters used for the solvents were: the simple point charge model (SPC/E) $)^{64}$ for water; the Bohm et al ${ }^{65}$ parameters for acetonitrile; and the OPLS-AA ${ }^{63}$ for dichloromethane and cyclohexane in the chair conformation.

The MC simulation consisted of a thermalization stage of $1.2 \times 10^{8} \mathrm{MC}$ steps, followed by an equilibrium stage of $1.5 \times 10^{8} \mathrm{MC}$ steps, where in each step one molecule was randomly selected to translate and rotate according to the Metropolis sampling technique. A thermalized configuration obtained from the MC simulation was used to start the MD simulations. A thermalization phase of 3 ns was performed to equilibrate the kinetic and potential energy and the MD simulation was carried out for more $6 \mathrm{~ns}$. The time step was 0.1 fs. To solve the equations of motion the integrator method was the velocity-Verlet. ${ }^{66}$ To keep the temperature and pressure constant, Berendsen thermostat and barostat were used. ${ }^{67}$ All the MC simulations were performed with the DICE program $^{68,69}$ and the MD was performed using the TINKER program. ${ }^{70,71}$

After the simulations, 100 statistically uncorrelated configurations of the solutesolvent system were selected and submitted to QM calculations of the absorption energies. The configurations selected for the QM calculation were composed by one Prodan molecule, the first solvation shell as explicit solvent molecules and the second solvation shell treated as an electrostatic embedding using the atomic charges of the solvent molecules (obtained from the classical force field). The calculations were first performed using the semi-empirical INDO-CIS method considering all occupied and unoccupied valence orbitals, i.e., a full CIS considering 43 occupied orbitals and 44 unoccupied. Another set of QM calculations was performed with 100 statistically uncorrelated configurations to generate ASEC where all solvent molecules are treated as an electrostatic embedding described by atomic point charges. 


\section{Results and Discussions}

\section{Geometry, polarization and solvation}

The optimized geometry of Prodan in the ground state $\mathrm{S}_{0}$ obtained with the B3LYP/6$31 \mathrm{G}(\mathrm{d})$ method is a planar structure with a small bending of the methyl groups bonded to the nitrogen atom $\left(\varphi_{\mathrm{C}-\mathrm{N}-\mathrm{CH}_{3}-\mathrm{CH}_{3}}=165.3^{\circ}\right)$ in good agreement with the X-ray crystallographic structure. ${ }^{30}$ The calculated value of the dipole moment is $5.8 \mathrm{D}$ in vacuum with MP2/aug-ccpVDZ (see Table 1). In the solvent environment, the electronic state relaxes due to the solvent interaction and the dipole moment increases to $6.1 \mathrm{D}, 7.7 \mathrm{D}, 8.0 \mathrm{D}$ and $10.2 \mathrm{D}$ in cyclohexane, dichloromethane, acetonitrile and water, respectively, as discussed before ${ }^{43}$ using the iterative-QM/MM procedure with MP2/aug-cc-pVDZ. The polarization of Prodan in solution increases with the solvent polarity and the effect of the water is remarkable with an increase of $176 \%$. Analyzing the charge distribution of the Prodan in vacuum (nonpolarization), there is a small charge separation between the electron donor and acceptor groups $\left(q_{i}(\mathrm{~N})-q_{i}(\mathrm{O})=0.16 e\right)$ but a large local charge separation in the $\mathrm{C}=\mathrm{O}$ bond $\left(q_{i}(\mathrm{C})-q_{i}(\mathrm{O})=0.80 \mathrm{e}\right)$. In solution, the charge separation between the electron donor and acceptor groups increases with the solvent polarity $\left(q_{i}(\mathrm{~N})-q_{i}(\mathrm{O})=0.25,0.30,0.26\right.$ and $0.47 e$, for cyclohexane, dichloromethane, acetonitrile and water, respectively), and the local charge separation in the $\mathrm{C}=\mathrm{O}$ bond increases even more $\left(q_{i}(\mathrm{C})-q_{i}(\mathrm{O})=0.99,1.10,1.06\right.$ and $1.36 e$, respectively). The electronic distribution of Prodan is very sensitive to the environment and hence subjected to considerable polarization.

The geometry of the ground state $\mathrm{S}_{0}$ obtained with CASSCF/ANO-L in vacuum is similar to the one optimized with the B3LYP/6-31G(d) method discussed above. The vertical excited states (in the $\mathrm{S}_{0}$ equilibrium geometry), $\pi-\pi^{*} \mathrm{~L}_{\mathrm{b}}, n-\pi^{*}$ and $\pi-\pi^{*} \mathrm{~L}_{\mathrm{a}}$ are close in energy, within $0.3 \mathrm{eV}\left(2.2 \times 10^{3} \mathrm{~cm}^{-1}\right)$, but they have quite different electronic structure that leads to the dipole moments of 5.7, 1.5 and 11.4D, respectively, in vacuum. Therefore, comparing their dipole moment with the ground state $\mathrm{S}_{0}(5.3 \mathrm{D})$, the $\pi-\pi^{*} \mathrm{~L}_{b}$ state has a similar value, the $n-\pi^{*}$ state has a much smaller value and the $\pi-\pi^{*} \mathrm{~L}_{\mathrm{a}}$ state has a much larger value. These three excited states relax to their equilibrium geometry stabilizing by $0.3,1.2$ and $0.7 \mathrm{eV}$, respectively. The geometry differences of the relaxed low-lying excited states in vacuum compared to the ground state are small, mostly the planarity of the amino group and the $\mathrm{C}=\mathrm{O}$ distance. The $\pi-\pi^{*} \mathrm{~L}_{\mathrm{b}}, n-\pi^{*}$ and $\pi-\pi^{*} \mathrm{~L}_{\mathrm{a}}$ equilibrium geometries are fully planar, i.e., with planar improper dihedral angle between the methyl groups bonded to the nitrogen atom and 
the aromatic rings, $\varphi_{C-N-C H_{3}-C H_{3}}=179^{\circ}$ for $\pi-\pi^{*} \mathrm{~L}_{\mathrm{b}}$ and $180^{\circ}$ for $n-\pi^{*}$ and $\pi-\pi * \mathrm{~L}_{\mathrm{a}}$. Moreover, the $\mathrm{C}=\mathrm{O}$ distances are $d_{C O}=1.225 \AA$ for $\mathrm{S}_{0}, 1.210 \AA$ for $\pi-\pi^{*} \mathrm{~L}_{\mathrm{b}} 1,1.357 \AA$ for $n-\pi^{*}$ and 1.216 $\AA$ for $\pi-\pi^{*} \mathrm{~L}_{\mathrm{a}}$. Additionally for the $n-\pi^{*}$ geometry, there are variations of the related angles, such as $\theta_{O-C-C H_{2}}=120^{\circ}$ for $\mathrm{S}_{0}, \pi-\pi^{*} \mathrm{~L}_{\mathrm{b}}$ and $\pi-\pi^{*} \mathrm{~L}_{\mathrm{a}}$ and $113^{\circ}$ for $n-\pi^{*}$. The main difference between the equilibrium geometry of the $\pi-\pi * \mathrm{~L}_{\mathrm{a}}$ and $\pi-\pi * \mathrm{~L}_{\mathrm{b}}$ is a small reduction in the distance of the nitrogen atom and the carbon of the rings, $d_{N C}=1.385 \AA$ for $\pi-\pi^{*} \mathrm{~L}_{\mathrm{a}}$ and 1.339 $\AA$ for $\pi-\pi^{*} \mathrm{~L}_{\mathrm{b}}$. All the Cartesian coordinates of the four equilibrium geometries $\left(\mathrm{S}_{0}, \pi-\pi^{*} \mathrm{~L}_{\mathrm{b}}, n\right.$ $\pi^{*}$ and $\pi-\pi^{*} \mathrm{~L}_{\mathrm{a}}$ ) are presented in the Supporting Information (SI).

The geometry optimizations and the minimum energy path (MEP) for the three lowlying excited states were performed also in geometries with rotations at the $-\mathrm{N}\left(\mathrm{CH}_{3}\right)_{2}$ and $\mathrm{COCH}_{2} \mathrm{CH}_{3}$ groups. But in vacuum the planar geometries were found to be the most stables, with lower energies.

Table 1. The atomic charges (in $e$ ) of some atoms, $\mathrm{C}=\mathrm{O}$ and $\mathrm{N}$, and the dipole moment (in D) of Prodan in vacuum and in aqueous solution are shown. The values are calculated for the ground state $\left(\mathrm{S}_{0}\right)$ and for the three low-lying relaxed excited states $\left(\pi-\pi^{*} \mathrm{~L}_{\mathrm{b}}, n-\pi^{*}\right.$ and $\left.\pi-\pi * \mathrm{~L}_{\mathrm{a}}\right)$ with CASSCF/ANO-L/ESPF. In parenthesis are shown the values for the vertical exited states. For comparison the atomic charges of the $\mathrm{S}_{0}$ were also calculated with MP2/aug-cc-pVDZ/CHELPG.

\begin{tabular}{cccccc}
\hline & $\begin{array}{c}\mathrm{S}_{0} \\
(\mathrm{MP} 2)\end{array}$ & $\begin{array}{c}\mathrm{S}_{0} \\
(\mathrm{CASSCF})\end{array}$ & $\begin{array}{c}\pi-\pi * \mathrm{~L}_{\mathrm{b}} \\
(\mathrm{CASSCF})\end{array}$ & $\begin{array}{c}n-\pi^{*} \\
(\mathrm{CASSCF})\end{array}$ & $\begin{array}{c}\pi-\pi^{*} \mathrm{~L}_{\mathrm{a}} \\
(\mathrm{CASSCF})\end{array}$ \\
\hline Vacuum & & & & & 0.04 \\
$q_{i}(\mathrm{C})$ & 0.36 & 0.34 & 0.48 & -0.15 & -0.52 \\
$q_{i}(\mathrm{O})$ & -0.44 & -0.42 & -0.59 & -0.03 & -0.03 \\
$q_{i}(\mathrm{~N})$ & -0.28 & -0.18 & -0.07 & $1.6(1.5)$ & $11.2(11.4)$ \\
$\mu$ & 5.8 & 5.3 & $5.8(5.7)$ & & 0.45 \\
\hline In water & & & & -0.10 & -0.89 \\
$q_{i}(\mathrm{C})$ & 0.60 & 0.86 & 0.66 & -0.22 & -0.17 \\
$q_{i}(\mathrm{O})$ & -0.76 & -0.88 & -0.87 & -0.21 & $17.2(17.9)$ \\
$q_{i}(\mathrm{~N})$ & -0.29 & -0.09 & -0.05 & $2.7(4.8)$ & $9.7(9.7)$ \\
$\mu$ & 10.2 & 9.1 & & & \\
\hline
\end{tabular}

Using the equilibrium geometry for each one of the four electronic states of Prodan, the charge distribution was calculated in vacuum and in aqueous solution with CASSCF/ANO-L/ESPF using the iterative-QM/MM procedure with ASEC. The calculated values of the dipole moment in vacuum are $\mu_{\mathrm{vac}}\left(\mathrm{S}_{0}\right)=5.3 \mathrm{D}, \mu_{\mathrm{vac}}\left(\pi-\pi^{*} \mathrm{~L}_{\mathrm{b}}\right)=5.8 \mathrm{D}, \mu_{\mathrm{vac}}\left(n-\pi^{*}\right)$ $=1.6 \mathrm{D}$ and $\mu_{\mathrm{vac}}\left(\pi-\pi * \mathrm{~L}_{\mathrm{a}}\right)=11.2 \mathrm{D}$ (see Table 1). Therefore, the relaxation to the equilibrium geometry has only a small effect in the dipole moment of the excited states compared with 
the vertically excited states (in the $\mathrm{S}_{0}$ geometry). In the other hand, the solvent effect is considerable. Their fully relaxed dipole moments in water are $\mu_{\mathrm{wat}}\left(\mathrm{S}_{0}\right)=9.1 \mathrm{D}, \mu_{\mathrm{wat}}\left(\pi-\pi^{*} \mathrm{~L}_{\mathrm{b}}\right)$ $=9.7 \mathrm{D}, \mu_{\mathrm{wat}}\left(n-\pi^{*}\right)=2.7$ and $\mu_{\mathrm{wat}}\left(\pi-\pi^{*} \mathrm{~L}_{\mathrm{a}}\right)=17.2 \mathrm{D}$. The electronic polarization of the Prodan in water is remarkable with an increase in the dipole moment of $160 \%$ in the $\mathrm{S}_{0}, 167 \%$ at $\pi$ $\pi^{*} \mathrm{~L}_{\mathrm{b}}, 181 \%$ in the $n-\pi^{*}$ and $154 \%$ in the $\pi-\pi^{*} \mathrm{~L}_{\mathrm{a}}$, considering the relaxed equilibrium geometry of each state optimized in vacuum. These dipole moment values are also presented in Table 1 together with some relevant atomic charges. The complete sets of atomic charges (in vacuum and polarized in water) are presented in the SI, together with the dipole moments evolutions during the iterative-QM/MM polarization procedure.

Comparing the ground state $\mathrm{S}_{0}$ with the $\pi-\pi^{*} \mathrm{~L}_{\mathrm{b}}, n-\pi^{*}$ and $\pi-\pi^{*} \mathrm{~L}_{\mathrm{a}}$ excited states, it was found that the $\pi-\pi^{*} L_{b}$ state has a dipole moment slightly larger than the $\mathrm{S}_{0}\left(\mu_{\mathrm{wat}}\left(\mathrm{S}_{0}\right)=9.1 \mathrm{D}\right.$ and $\left.\mu_{\mathrm{wat}}\left(\pi-\pi^{*} \mathrm{~L}_{\mathrm{b}}\right)=9.7 \mathrm{D}\right)$, but the $n-\pi^{*}$ state has a large decrease $\left(\mu_{\mathrm{wat}}\left(n-\pi^{*}\right)=2.7 \mathrm{D}\right)$, and the $\pi-\pi * \mathrm{~L}_{\mathrm{a}}$ state has a large increase $\left(\mu_{\mathrm{wat}}\left(\pi-\pi * \mathrm{~L}_{\mathrm{a}}\right)=17.2 \mathrm{D}\right)$.

Recently, Baral et $a l^{72}$ studied the relaxation of the first excited state $\mathrm{S}_{1}$ $(\mathrm{HOMO} \rightarrow \mathrm{LUMO})$ of Prodan in several solvents (hexane, acetone, octanol, ethanol, methanol and water) using theoretical procedure and ultrafast time-resolved transient absorption measurements. Comparing the Prodan molecular orbital calculated by them and by us, we identified that the reported $\mathrm{S}_{1}$ state is the $\pi-\pi^{*} \mathrm{~L}_{\mathrm{a}}$ state (see discussion about the excited states in the next section). They used an iterative-QM/MM procedure, similar to ours, where the QM calculations are performed with many-body Green functions within the GW approximation and the Bethe-Salpeter equation (GW-BSE). The MM simulations were made with the MD simulations, recalculating the atomic charges of the Prodan at the $\mathrm{S}_{1}$ state with electrostatic embedding in each iterative step. In hexane, they obtained the vertical nonrelaxed dipole moment of $5.50 \mathrm{D}$ and the fully relaxed $\mu_{\text {hex }}\left(\mathrm{S}_{1}\right)=10.98 \mathrm{D}$, and in water the vertical non-relaxed $\sim 9 \mathrm{D}$ and the fully relaxed $\mu_{\text {hex }}\left(\mathrm{S}_{1}\right) \sim 24 \mathrm{D}$, showing a remarkable polarization and relaxation effects of the Prodan $S_{1}$ around $200 \%$ in hexane and $266 \%$ in water. These values are larger than our calculated values and the reason may come from two differences: (i) the QM methods are different, which can underestimate or overestimate the solvent interaction with the solute wavefunction; and (ii) we performed MM simulation of Prodan in solution with rigid geometry and Baral et al. ${ }^{72}$ used flexible Prodan geometry, considering the geometry relaxation due to the solvent interactions. But since they used the same force field bonded parameters for the ground state $S_{0}$ and the first excited state $S_{1}$, a 
similar geometry of both states is implicitly assumed and this is in qualitative agreement with the $\pi-\pi * \mathrm{~L}_{\mathrm{a}}$ geometry obtained in this work.

The solvation shells around Prodan is determined using the minimum distance distribution function (MDDF) $)^{73,74}$ and the effect of the polarization of the solute due to the solvent is analyzed. The MDDFs between Prodan in the ground state $\mathrm{S}_{0}$ and the studied solvents are presented in the SI. For the solvents cyclohexane, dichloromethane and acetonitrile, no significant changes were observed in the average solvation shells using the non-polarized and polarized sets of atomic charges of Prodan using MP2/aug-ccpVDZ/CHELPG. However, for the aqueous solution, although the number of water molecules in the solvation shells remains the same, an increase in the number of hydrogen bonds was observed. The quantity of solvent molecules in the first and second solvation shells was 20 (up to $4.15 \AA$ ) and 77 (from 4.15 to $9.35 \AA$ ) for cyclohexane, 24 (up to $4.65 \AA$ ) and 80 (from 4.65 to $8.55 \AA$ ) for dichloromethane, 29 (up to $4.15 \AA$ ) and 100 (from 4.15 to $7.95 \AA$ ) for acetonitrile and 56 (up to $4.05 \AA$ ) and 231 (from 4.05 to $7.95 \AA$ ) for water. For the polarized Prodan in water, it is possible to observe a small peak in the MDDF around 1.70 $\AA$ that is characteristic of hydrogen bonds (HBs) between Prodan and water molecules. The number of water molecules in the solvation shells is still the same for the four electronic states, $\mathrm{S}_{0}, \mathrm{~S}_{1}, \mathrm{~S}_{2}$ and $\mathrm{S}_{3}$, but the number of HBs differs.

\section{Absorption spectra}

The experimental absorption spectra of the fluorophore Prodan and Laurdan in the six solvents are presented in SI. In Fig. 3 only the first absorption band is shown along with the calculated excitation energies. As it can be seen this band is very broad in all solvents. Therefore, when referring to the experimental value one should consider that this is the band maximum of a considerably broad absorption band.

The results obtained with INDO-CIS/SCRF and TD-B3LYP/PCM for the first three excitations are presented as vertical lines in Fig. 3. For the INDO-CIS/Explicit the excitations were calculated for 100 solute-solvent configurations obtained from the simulations. This leads naturally to band broadening and the three calculated excitations are presented in Fig. 3 as histograms, giving a very good description of the observed band. In the case of water the solute-solvent configurations were generated with two simulation methods, MC and MD. The difference between them is the flexibility of the solute. As it can be seen in Fig. 3, the outcome of 100 QM calculations with the INDO-CIS/Explicit using solute-solvent configurations obtained from MC simulation (with rigid solute) present a broadening caused 
by the diversity of solvent positions around the solute and in the case of MD simulation (with flexibly solute) presents an even broader distribution of excitation energy due to the solute deformation. This describes very well the broadening of this first absorption band in water. Analyzing the Prodan conformations during the MD simulation, the planarity was maintained with rotational angles variations between the aromatic rings and the $-\mathrm{N}\left(\mathrm{CH}_{3}\right)_{2}$ and the $\mathrm{COCH}_{2} \mathrm{CH}_{3}$ groups around $\pm 15^{\circ}$, but mostly the deformation comes from the $\mathrm{C}=\mathrm{O}$ stretch caused by the hydrogen bond formed between the Prodan and the water molecules. In cyclohexane, dichloromethane and acetonitrile we see the separation of the first three excitations (solid histogram in red for the $n-\pi^{*}$ and in blue for the $\pi-\pi^{*}$ ) of 100 solute-solvent configurations obtained with MC simulation.

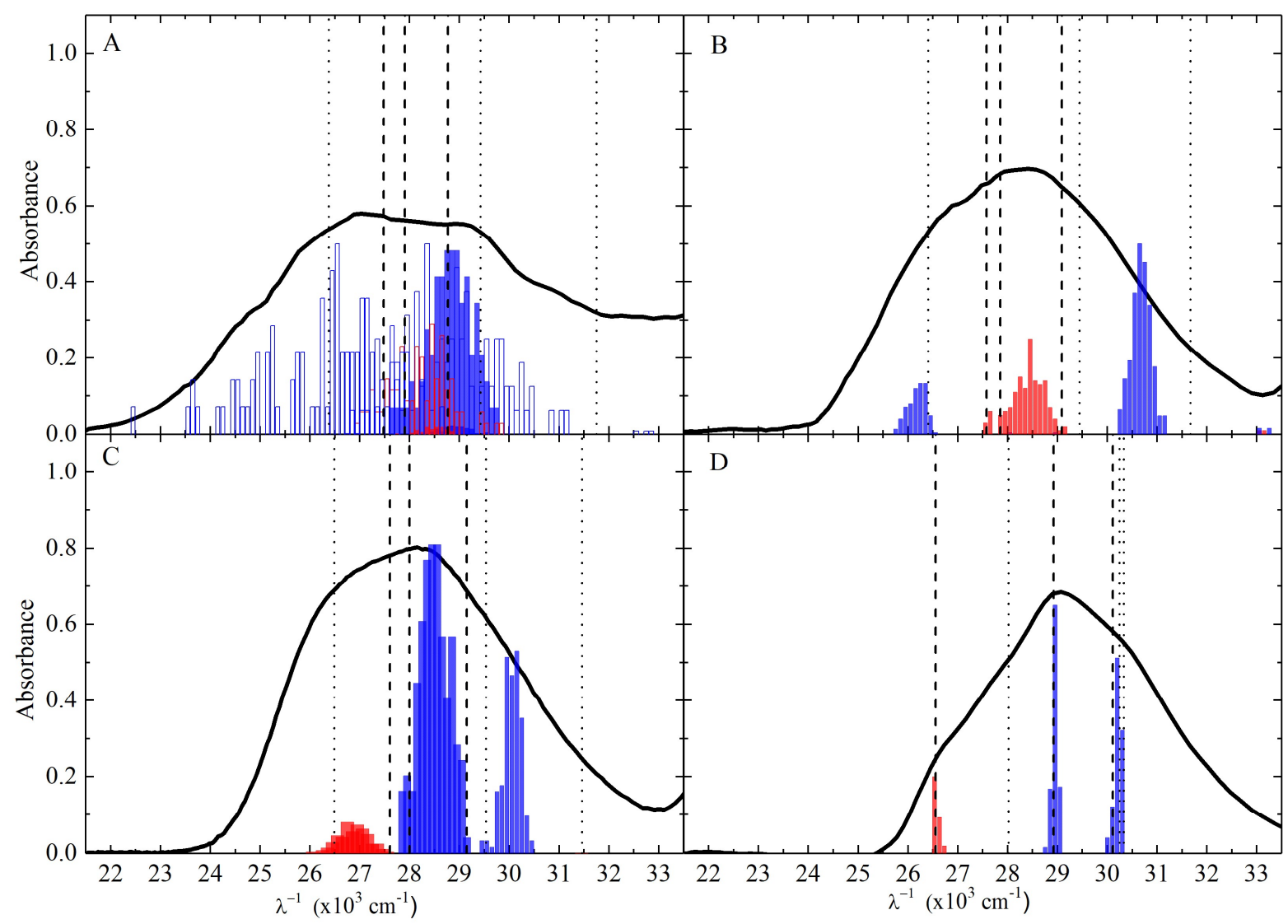

Figure 3: Absorption spectra of Prodan in several solvents: (A) in water, (B) in acetonitrile, (C) in dichloromethane and (D) in cyclohexane. Black solid line is the experimental result (this work). Vertical lines are the first three excitations calculated with INDO-CIS/SCRF (dashed) and with B3LYP/PCM (dotted). The histograms (in red is $n-\pi^{*}$ and in blue is $\pi-\pi^{*}$ excitations) are the excitations calculated with INDO-CIS/Explicit for 100 different solute-solvent configurations obtained with MC simulations for all solvent (solid histogram) and with MD simulation (open histogram) only for water.

All methods used in this work agree that the first experimental band is composed of three excitations: one $n-\pi^{*}$ and the two $\pi-\pi^{*}$ transitions. In nonpolar environments (vacuum, 
cyclohexane and dichloromethane), the INDO-CIS/Explicit, INDO-CIS/SCRF and the TDB3LYP/PCM calculations show the $n-\pi^{*}$ as the first transition followed by the two $\pi-\pi^{*}$. In polar solvents (acetonitrile and water), the $n-\pi^{*}$ transition becomes the second excited state. This is easy to understand because in more polar solvents the $n-\pi^{*}$ transition shifts to the blue (higher energy) whereas the $\pi-\pi *$ transitions shift to the red (lower energy). This is summarized in Table 2. The experimental excitation energies (and wavelengths) presented at Table 2 correspond to the maximum absorption. However, to compare with the theoretical results it is important to consider the large broadening of this band as seen in Fig. 3.

Table 2: Calculated vertical excitation energies for Prodan in vacuum and in different solvents calculated with four different methods. In the case of explicit solvent, the values were obtained with average over 100 solutesolvent configurations selected from MC simulations for all solvents except for water that is from MD simulation. The values are in $10^{3} \mathrm{~cm}^{-1}$ and in parentheses in $\mathrm{nm}$. The experimental values are the energy of the band maximum shown in Fig. 3 and obtained in this work. The asterisk indicates $n-\pi^{*}$ transition, in brackets indicate the CASPT2/ASEC method and in boldface is the best agreement with the experimental energy of maximum absorption $\left(\mathrm{E}_{\mathrm{abs}}{ }^{\mathrm{exp}}\right)$. The experimental values ${ }^{10}$ for the higher emission energy $\mathrm{E}_{1}{ }^{\text {exp }}$, lower emission energy $\mathrm{E}_{2}{ }^{\exp }$ are shown and the Stokes shift $\left(\Delta \mathrm{E}_{1}{ }^{\exp }(\mathrm{SS})=\mathrm{E}_{1}{ }^{\exp }-\mathrm{E}_{\mathrm{abs}}{ }^{\exp }\right.$ and $\left.\Delta \mathrm{E}_{2}{ }^{\exp }(\mathrm{SS})=\mathrm{E}_{2}{ }^{\exp }-\mathrm{E}_{\mathrm{abs}}{ }^{\exp }\right)$ are also shown.

\begin{tabular}{|c|c|c|c|c|c|}
\hline Excitation & Vacuum & Cyclohexane & Dichloromethane & Acetonitrile & Water \\
\hline \multicolumn{6}{|c|}{ Method: INDO-CIS/Explicit } \\
\hline 1st & $26.7(375)^{*}$ & $26.6 \pm 0.1(376)^{*}$ & $26.9 \pm 0.3(372)^{*}$ & $26.2 \pm 0.3(382)$ & $27.2 \pm 1.2(368)$ \\
\hline 2nd & $29.1(343)$ & $29.0 \pm 0.1(345)$ & $28.5 \pm 0.5(351)$ & $28.5 \pm 0.5(351) *$ & $28.5 \pm 0.7(351)^{*}$ \\
\hline 3rd & $30.0(333)$ & $30.2 \pm 0.1(331)$ & $30.0 \pm 0.3(333)$ & $30.7 \pm 0.3(326)$ & $28.5 \pm 1.1(351)$ \\
\hline \multicolumn{6}{|c|}{ Method: INDO-CIS/SCRF } \\
\hline $1 \mathrm{st}$ & $26.7(375)^{*}$ & $26.9(372)^{*}$ & $27.6(362)^{*}$ & $27.6(363)$ & $27.5(364)$ \\
\hline 2nd & $29.1(343)$ & $28.8(347)$ & $28.0(357)$ & $27.9(359)^{*}$ & $27.9(358)^{*}$ \\
\hline 3rd & $30.0(333)$ & $30.0(333)$ & $29.1(343)$ & $29.1(344)$ & $28.8(348)$ \\
\hline \multicolumn{6}{|c|}{ Method: TD-B3LYP/PCM } \\
\hline 1st & $28.0(370)^{*}$ & $27.1(370)^{*}$ & $26.5(377)^{*}$ & $26.4(379)$ & $26.4(379)$ \\
\hline 2nd & $30.3(331)$ & $29.9(334)$ & $29.5(339)$ & $29.4(340)^{*}$ & $29.4(340)^{*}$ \\
\hline 3 rd & $30.3(330)$ & $30.8(325)$ & $31.5(318)$ & $32.0(313)$ & $31.7(316)$ \\
\hline \multicolumn{6}{|c|}{ Method: CASPT2/PCM[ASEC] } \\
\hline $1 \mathrm{st}$ & $31.9(313)$ & - & - & - & $30.6(327)[30.4]$ \\
\hline 2nd & $32.0(313)^{*}$ & - & - & - & $31.9(313)[31.3]$ \\
\hline 3rd & $34.4(291)$ & - & - & - & $33.6(298) *[31.8]$ \\
\hline \multicolumn{2}{|c|}{ Absorption energy $E_{a b s} \exp$} & $29.1(344)$ & $28.2(354)$ & $28.2(354)$ & $27.6(362)$ \\
\hline \multicolumn{2}{|c|}{ High energy emission $\mathrm{E}_{1} \exp$} & $25.5(393)$ & $22.9(437)$ & $21.4(467)$ & $19.1(522)$ \\
\hline \multicolumn{2}{|c|}{ Low energy emission $E_{2} \exp$} & $24.1(415)$ & $21.5(466)$ & $20.3(493)$ & $17.3(579)$ \\
\hline \multicolumn{2}{|c|}{ Stokes shift $\Delta \mathrm{E}_{1}{ }^{\exp }(\mathrm{SS})$} & -3.6 & -5.3 & -6.8 & -8.5 \\
\hline \multicolumn{2}{|c|}{ Stokes shift $\Delta \mathrm{E}_{2}{ }^{\exp }(\mathrm{SS})$} & -5.0 & -6.7 & -7.9 & -10.3 \\
\hline
\end{tabular}

The excitation energies (Table 2) calculated with CASPT2 are larger than those calculated with B3LYP or the two INDO-CIS models and also the experimental data. Comparing CASPT2/ASEC with B3LYP/PCM or INDO-CIS/Explicit, the first $\pi$ - $\pi^{*}$ excitation in water differs by 4.0 or $3.2 \times 10^{3} \mathrm{~cm}^{-1}$, respectively. In changing from vacuum to water using the CASPT2/ASEC method, the solvent shift in absorption is $-1.5 \times 10^{3} \mathrm{~cm}^{-1}$ for the first $\pi-\pi^{*}$ excitation, $-0.7 \times 10^{3} \mathrm{~cm}^{-1}$ for the $n-\pi^{*}$ excitation and $-2.6 \times 10^{3} \mathrm{~cm}^{-1}$ for the 
second $\pi-\pi^{*}$ excitation. The experimental solvent shift going from an nonpolar solvent (cyclohexane) to a polar solvent (water) is $-1.5 \times 10^{3} \mathrm{~cm}^{-1}$. Therefore, the solvent shift of the first $\pi-\pi^{*}$ excitation represents the best agreement between the CASPT2/ASEC method and the experimental data. Theses values are comparable to the shifts obtained with the other theoretical models, shown in Table 2. In spite of obtaining large excitation energies the results at the CASPT2 level agree that the first observed band should be composed of three low-lying transitions. The separation between these three transitions amount to $3.0 \times 10^{3} \mathrm{~cm}^{-1}$ and is still much less that the broadening experimentally observed to the first absorption band. Then, we conclude that all theoretical models adopted here agree that the first absorption band of Prodan in solution is composed by vertical excitations from the ground state to three different excited states, one $n-\pi^{*}$ and two $\pi-\pi^{*}$ states where the first $\pi$ - $\pi^{*}$ excitation represents the best agreement between theoretical and experimental solvent shift.

There are several previous studies that report theoretical results of the absorption energies of Prodan in vacuum..$^{20,22,30,31,75}$ Most of these results underestimate the first transition and overestimate the second when compared with the results in Table 2. Two previous papers, ${ }^{19,36}$ using the PCM model for the solvent obtained results that are in good agreement with the experimental data. Therefore, considering Fig. 3 and Table 2, it is possible to conclude that the absorption spectra of Prodan in all the solvents considered are well described by three electronic excitations.

\section{The excited electronic states and the emission spectrum}

To analyze the excited states of Prodan we use the more adequate CASSCF and CASPT2 methods. The low-lying excited electronic states were first identified from the absorption transitions computed at the CASPT2 level in vacuum. Three relevant excited electronic states were identified. The $\pi-\pi^{*} \mathrm{~L}_{\mathrm{b}}$ state is the first $\pi-\pi^{*}$ electronic state in vacuum mainly described by the transitions HOMO $-1 \rightarrow$ LUMO and HOMO $\rightarrow$ LUMO +1 , while $n-\pi^{*}$ state is characterized by the transition from the oxygen lone-pair orbital (HOMO-3) to the LUMO +1 and $\pi-\pi * \mathrm{~L}_{b}$ state is mainly described by the HOMO $\rightarrow$ LUMO transition. The molecular orbitals characterizing these electronic states are shown in Fig. 4. These orbitals are in good agreement with those obtained by Baral et al. ${ }^{72}$ using QM calculations with GWBSE level. 


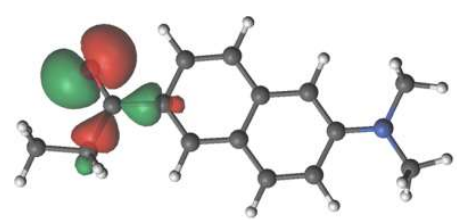

HOMO-3

(Lone-pair)

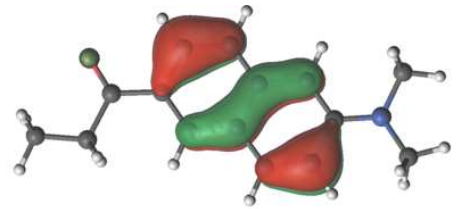

HOMO-1

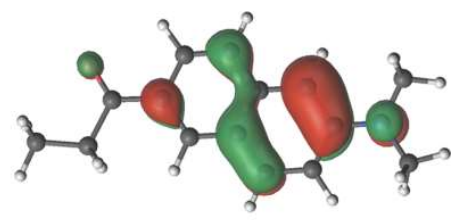

HOMO

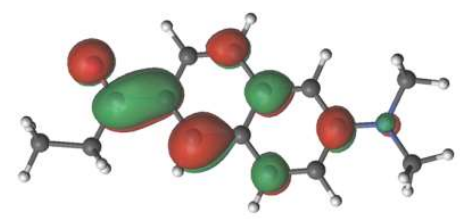

LUMO

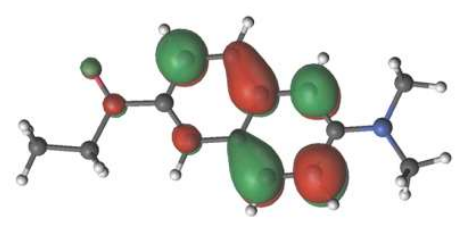

LUMO+1

Figure 4. Molecular orbitals involved in the electron transition of the three lowest excited electronic states: $\pi$ $\pi * \mathrm{~L}_{\mathrm{a}}$ is dominated by HOMO $\rightarrow$ LUMO transition; $\pi-\pi * \mathrm{~L}_{\mathrm{b}}$ is dominated by a combination of HOMO $\rightarrow$ LUMO +1 and HOMO- $1 \rightarrow$ LUMO transitions; and $n-\pi *$ is dominated by HOMO- $3 \rightarrow$ LUMO +1 transition.

To better analyze the photophysics of Prodan in vacuum and in water solution, the energy of the ground and three excited electronic states were recalculated with CASPT2 level using the equilibrium geometries obtained with $\operatorname{CASSCF}(12,12)$ level in vacuum. Therefore, sixteen CASPT2 calculations were performed to obtain the electronic energies of four electronic states $\left(\mathrm{S}_{0}, \pi-\pi^{*} \mathrm{~L}_{\mathrm{b}}, n-\pi^{*}\right.$ and $\left.\pi-\pi^{*} \mathrm{~L}_{\mathrm{a}}\right)$ in the equilibrium geometry of each four state obtained with CASSCF. This method will be called CASPT2//CASSCF. The energy differences (corrected by zero-point energy and vibrational entropy) from the ground state $\mathrm{S}_{0}$ to the excited states $\pi-\pi^{*} \mathrm{~L}_{\mathrm{b}}, n-\pi *$ and $\pi-\pi * \mathrm{~L}_{\mathrm{a}}$ are presented in Table 3, and these energy values were used to make a schematic representation of the Prodan photophysics shown in Fig. 5, in vacuum (A) and in aqueous solution (B). In the x-axis, the geometries were ordered in the sequence $n-\pi^{*}, \pi-\pi^{*} \mathrm{~L}_{\mathrm{b}}, \mathrm{S}_{0}$ and $\pi-\pi^{*} \mathrm{~L}_{\mathrm{a}}$ to describe the symbolic reaction coordinates. This order is arbitrary but was selected in the way to reproduce a well-behaved minimum energy profile of the ground state. In Fig. 5, the photophysics of Prodan is schematically summarized including some important energy differences presented at Table 3. The grey region indicated in Fig. 5A shows an important characteristic of this molecular system, since excitation energies from about 31.9 to $34.2 \times 10^{3} \mathrm{~cm}^{-1}(292-313 \mathrm{~nm})$ could populate the first three excited electronic states, as they are very close in energy. In water, the excitation energies range from about 30.4 to $31.8 \times 10^{3} \mathrm{~cm}^{-1}(329-314 \mathrm{~nm})$ also could populate the first three excited electronic states.

Taking into account that the $n-\pi^{*}$ excited state is a dark state due to the weak oscillator strength of the electronic transition, it is expected that the $\pi-\pi^{*} L_{b}$ and $\pi-\pi^{*} L_{a}$ states 
are the main populated states upon the Franck-Condon absorption transition. Following the minimum energy path (MEP) of these three states in vacuum and in water, it can be observed that internal conversions may occur. These possible internal conversions between excited states of Prodan were not investigated in this work, but it shows clearly that the study of only $\mathrm{S}_{1}$ state is not enough to describe the complex dual fluorescence of Prodan in solution and even more complex in heterogeneous environment such as phospholipid membranes.

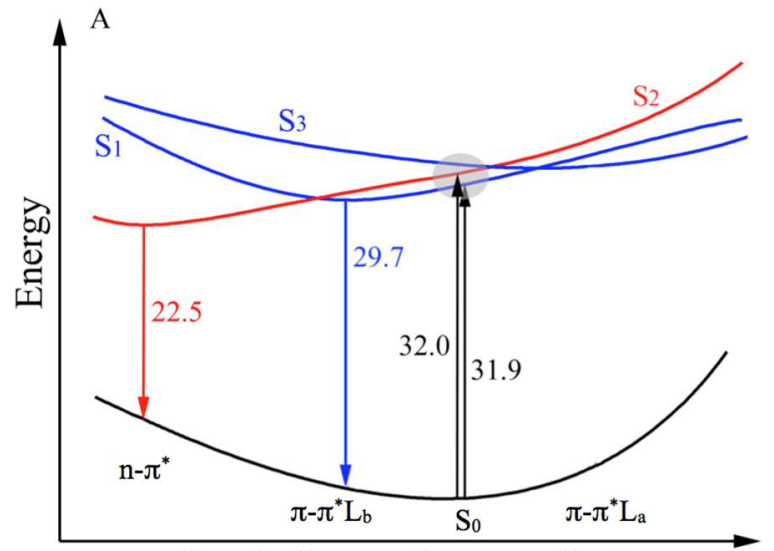

Symbolic reaction coordinates

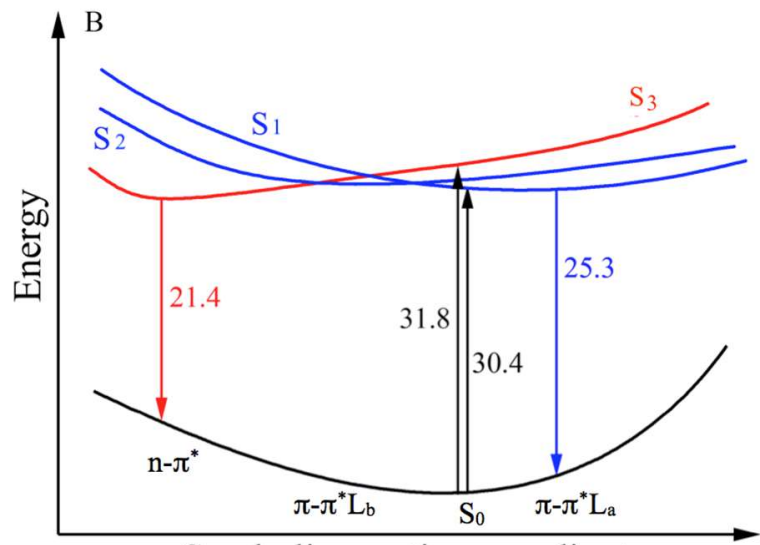

Symbolic reaction coordinates

Figure 5. Schematic representation of the photophysics of the Prodan in vacuum (A) and in water (B) using CASPT2//CASSCF level with the solvent described by the ASEC electrostatic embedding. The energy is the total internal energy corrected by zero-point energy and vibrational entropy. The $\pi-\pi^{*}$ states are represented in blue and the $n-\pi^{*}$ state in red. The values (in $\times 10^{3} \mathrm{~cm}^{-1}$ ) were presented in Table 3 .

The $n-\pi^{*}$ state is the lowest excited state at its corresponding equilibrium geometry in vacuum and in water. The calculated emission transition energy $\mathrm{S}_{3} \rightarrow \mathrm{S}_{0}$ in water is $21.4 \times 10^{3}$ $\mathrm{cm}^{-1}$, that is $4.1 \times 10^{3} \mathrm{~cm}^{-1}$ larger than the experimental lower energy emission $\mathrm{E}_{2}{ }^{\exp }=17.3 \mathrm{x}$ $10^{3} \mathrm{~cm}^{-1}$ in water (see Table 2). For the absorption transition energy of the $\pi-\pi^{*} \mathrm{~L}_{\mathrm{a}}$ state, the calculated value with CASPT2/ASEC method, $\mathrm{E}_{\mathrm{abs}}{ }^{\mathrm{cal}}=30.4 \times 10^{3} \mathrm{~cm}^{-1}$, is also overestimated compared with the experimental data, $\mathrm{E}_{\mathrm{abs}}{ }^{\exp }=27.6 \times 10^{3} \mathrm{~cm}^{-1}$ (see Table 2). Therefore, the calculated Stokes shift of the lower energy $\Delta \mathrm{E}_{2}{ }^{\mathrm{cal}}(\mathrm{SS})=-9.0(21.4-30.4) \times 10^{3} \mathrm{~cm}^{-1}$ is in good agreement with the experimental value $\Delta \mathrm{E}_{2}{ }^{\exp }(\mathrm{SS})=-10.3 \times 10^{3} \mathrm{~cm}^{-1}$, as shown in Table 3.

The $\mathrm{S}_{1}$ state is the second lowest excited states of Prodan in vacuum and in water. However, in vacuum it is the $\pi-\pi^{*} \mathrm{~L}_{\mathrm{b}}$ state and in water it changes to the $\pi-\pi^{*} \mathrm{~L}_{\mathrm{a}}$ state. In water, the calculated emission transition energy $\mathrm{S}_{1} \rightarrow \mathrm{S}_{0}$ is $25.3 \times 10^{3} \mathrm{~cm}^{-1}$. This energy is also overestimated in comparison with the experimental higher energy emission in water, $\mathrm{E}_{1}{ }^{\exp }=$ $19.1 \times 10^{3} \mathrm{~cm}^{-1}$ (see Table 2). The calculated value for the Stokes shift of the higher energy 
are $\Delta \mathrm{E}_{1}{ }^{\mathrm{cal}}(\mathrm{SS})=-5.1(=25.3-30.4) \times 10^{3} \mathrm{~cm}^{-1}$ is in agreement with the experimental Stokes shift in water, $\Delta \mathrm{E}_{1}{ }^{\exp }(\mathrm{SS})=-8.5 \times 10^{3} \mathrm{~cm}^{-1}$, as shown in Table 3 .

Table 3. Transition energies (in $10^{3} \mathrm{~cm}^{-1}$ ) of Prodan calculated at the CASPT2//CASSCF level in vacuum and in water using PCM and ASEC electrostatic embedding obtained with MC simulations. In boldface, the absorption transition energy $\left(\mathrm{S}_{0}-\mathrm{S}_{1}\right)$ and the emission transition energy $\left(\mathrm{S}_{1}-\mathrm{S}_{0}, \mathrm{~S}_{2}-\mathrm{S}_{0}\right.$ and $\left.\mathrm{S}_{3}-\mathrm{S}_{0}\right)$ were highlighted. The calculated Stokes shift $\left(\Delta \mathrm{E}_{1}{ }^{\text {cal }}(\mathrm{SS})=\mathrm{E}_{1}{ }^{\exp }-\mathrm{E}_{\mathrm{abs}}{ }^{\exp }\right.$ and $\left.\Delta \mathrm{E}_{2}{ }^{\mathrm{cal}}(\mathrm{SS})=\mathrm{E}_{2}{ }^{\exp }-\mathrm{E}_{\mathrm{abs}}{ }^{\mathrm{exp}}\right)$ are also shown in comparison with the experimental data presented at Table 2.

\begin{tabular}{|c|c|c|c|}
\hline & Vacuum & PCM (water) & ASEC (water) \\
\hline $\begin{array}{c}\text { Franck Condon } \mathrm{S}_{0} \text { geometry } \\
\pi-\pi * \mathrm{~L}_{\mathrm{b}} \\
n-\pi * \\
\pi-\pi * \mathrm{~L}_{\mathrm{a}} \\
\end{array}$ & $\begin{array}{l}\text { 31.9 } \mathrm{S}_{\mathbf{0}}-\mathrm{S}_{1} \\
32.0 \mathrm{~S}_{0}-\mathrm{S}_{2} \\
34.2 \mathrm{~S}_{0}-\mathrm{S}_{3} \\
\end{array}$ & $\begin{array}{l}31.9 \mathrm{~S}_{0}-\mathrm{S}_{2} \\
33.6 \mathrm{~S}_{0}-\mathrm{S}_{3} \\
\text { 30.6 } \mathrm{S}_{\mathbf{0}}-\mathrm{S}_{\mathbf{1}} \\
\end{array}$ & $\begin{array}{l}31.3 \mathrm{~S}_{0}-\mathrm{S}_{2} \\
31.8 \mathrm{~S}_{0}-\mathrm{S}_{3} \\
\mathbf{3 0 . 4} \mathbf{S}_{\mathbf{0}}-\mathrm{S}_{\mathbf{1}} \\
\end{array}$ \\
\hline $\begin{array}{c}\pi-\pi * \mathrm{~L}_{\mathrm{b}} \text { equilibrium geometry } \\
\pi-\pi * \mathrm{~L}_{\mathrm{b}} \\
n-\pi * \\
\pi-\pi * \mathrm{~L}_{\mathrm{a}}\end{array}$ & $\begin{array}{l}\text { 29.7 } \mathrm{S}_{\mathbf{1}}-\mathrm{S}_{\mathbf{0}} \\
29.9 \mathrm{~S}_{2}-\mathrm{S}_{0} \\
33.5 \mathrm{~S}_{3}-\mathrm{S}_{0}\end{array}$ & $\begin{array}{l}29.3 \mathrm{~S}_{2}-\mathrm{S}_{\mathbf{0}} \\
31.5 \mathrm{~S}_{1}-\mathrm{S}_{0} \\
30.8 \mathrm{~S}_{3}-\mathrm{S}_{0}\end{array}$ & $\begin{array}{l}\text { 29.3 } \mathbf{S}_{\mathbf{2}}-\mathrm{S}_{\mathbf{0}} \\
32.4 \mathrm{~S}_{1}-\mathrm{S}_{0} \\
30.1 \mathrm{~S}_{3}-\mathrm{S}_{0}\end{array}$ \\
\hline $\begin{array}{c}n-\pi \text { equilibrium geometry } \\
n-\pi^{*} \\
\pi-\pi * \mathrm{~L}_{\mathrm{b}} \\
\pi-\pi * \mathrm{~L}_{\mathrm{a}} \\
\end{array}$ & $\begin{array}{l}\text { 22.5 } \mathbf{S}_{\mathbf{2}}-\mathrm{S}_{\mathbf{0}} \\
30.4 \mathrm{~S}_{1}-\mathrm{S}_{0} \\
31.5 \mathrm{~S}_{3}-\mathrm{S}_{0} \\
\end{array}$ & $\begin{array}{l}\mathbf{2 4 . 8} \mathrm{S}_{\mathbf{3}}-\mathrm{S}_{\mathbf{0}} \\
30.4 \mathrm{~S}_{2}-\mathrm{S}_{0} \\
27.5 \mathrm{~S}_{1}-\mathrm{S}_{0} \\
\end{array}$ & $\begin{array}{l}21.4 \mathrm{~S}_{3}-\mathrm{S}_{\mathbf{0}} \\
31.1 \mathrm{~S}_{2}-\mathrm{S}_{0} \\
36.1 \mathrm{~S}_{1}-\mathrm{S}_{0} \\
\end{array}$ \\
\hline $\begin{array}{c}\pi-\pi * \mathrm{~L}_{\mathrm{a}} \text { equilibrium geometry } \\
\pi-\pi * \mathrm{~L}_{\mathrm{a}} \\
\pi-\pi * \mathrm{~L}_{\mathrm{b}} \\
n-\pi *\end{array}$ & $\begin{array}{l}\text { 30.6 } \mathbf{S}_{\mathbf{3}}-\mathrm{S}_{\mathbf{0}} \\
29.2 \mathrm{~S}_{1}-\mathrm{S}_{0} \\
31.7 \mathrm{~S}_{2}-\mathrm{S}_{0} \\
\end{array}$ & $\begin{array}{l}\text { 27.3 } \mathrm{S}_{\mathbf{1}}-\mathrm{S}_{\mathbf{0}} \\
30.3 \mathrm{~S}_{2}-\mathrm{S}_{0} \\
31.7 \mathrm{~S}_{3}-\mathrm{S}_{0} \\
\end{array}$ & $\begin{array}{l}\text { 25.3 } \mathrm{S}_{\mathbf{1}}-\mathrm{S}_{\mathbf{0}} \\
26.0 \mathrm{~S}_{2}-\mathrm{S}_{0} \\
33.0 \mathrm{~S}_{3}-\mathrm{S}_{0} \\
\end{array}$ \\
\hline $\begin{array}{l}\text { Stokes Shift of high energy } \\
\Delta \mathrm{E}_{1}{ }^{\text {cal }}(\mathrm{SS}) \\
\Delta \mathrm{E}_{1}{ }^{\exp }(\mathrm{SS}) \\
\text { Stokes Shift of low energy } \\
\Delta \mathrm{E}_{2}{ }^{\text {cal }}(\mathrm{SS}) \\
\Delta \mathrm{E}_{2}{ }^{\exp }(\mathrm{SS})\end{array}$ & $\begin{array}{c}-2.2 \mathrm{~S}_{1}-\mathrm{S}_{0}\left(\pi-\pi^{*} \mathrm{~L}_{\mathrm{b}}\right) \\
-3.6\end{array}$ & $\begin{array}{c}-3.3 \mathrm{~S}_{2}-\mathrm{S}_{0}\left(\pi-\pi * \mathrm{~L}_{\mathrm{a}}\right) \\
-8.5\end{array}$ & $\begin{array}{c}-5.1 \mathrm{~S}_{1}-\mathrm{S}_{0}\left(\pi-\pi * \mathrm{~L}_{\mathrm{a}}\right) \\
-8.5\end{array}$ \\
\hline
\end{tabular}

The calculated values for the Stokes shifts of the high and low energies of Prodan in vacuum, $\Delta \mathrm{E}_{1}{ }^{\text {cal }}(\mathrm{SS})=-2.2 \times 10^{3} \mathrm{~cm}^{-1}$ and $\Delta \mathrm{E}_{2}{ }^{\text {cal }}(\mathrm{SS})=-9.4 \times 10^{3} \mathrm{~cm}^{-1}$ (Table 3), were compared with the experimental measurements of the absorption transition and the dual fluorescent of Prodan in cyclohexane (Table 2 and 3 ), $\Delta \mathrm{E}_{1}{ }^{\exp }(\mathrm{SS})=-3.6 \times 10^{3} \mathrm{~cm}^{-1}$ and $\Delta \mathrm{E}_{2}{ }^{\exp }(\mathrm{SS})=-5.0 \times 10^{3} \mathrm{~cm}^{-1}$. Both Stokes shifts are for the red side and have a qualitative agreement showing a smaller shift for the high energy than for the low energy. For the low energy Stokes shift, our results are overestimated compared with the experimental data, probably because we are not considering the non-equilibrium solvation situation. The nonequilibrium solvation, describes the situation when the solute is in the excited state, but the solvent molecules had not enough time to relax their distribution around the solute. Usually, calculations on this situation show a smaller Stokes shift due to the larger similarity of the solvated ground state and the non-relaxed solvated excited state. Additionally, the lifetime decays for Prodan in solution are large, in nanoseconds scale, showing the major contribution of the fully relaxed solvation situation in its fluorescent emission spectra (experimental 
values $^{10}$ of $\tau_{1}=0.2 \mathrm{~ns}$ and $\tau_{2}=0.8 \mathrm{~ns}$ in cyclohexane and between 2.1-4.5ns for several solvents, such as chloroform, dichloromethane, acetonitrile, methanol and water). So, this non-equilibrium solvation effect is important and should be considered for a better description of the dual fluorescence of Prodan in low polarity solvents.

In the case of Prodan in water, the Stokes shifts of the high and low energies using the ASEC electrostatic embedding model are $\Delta \mathrm{E}_{1}{ }^{\mathrm{cal}}(\mathrm{SS})=-5.1 \times 10^{3} \mathrm{~cm}^{-1}$ and $\Delta \mathrm{E}_{2}{ }^{\mathrm{cal}}(\mathrm{SS})=-9.0 \mathrm{x}$ $10^{3} \mathrm{~cm}^{-1}$, showing that the solvent effect is better described by the ASEC than the PCM, $\Delta \mathrm{E}_{1}{ }^{\mathrm{cal}}(\mathrm{SS})=-3.3 \times 10^{3} \mathrm{~cm}^{-1}$ and $\Delta \mathrm{E}_{2}{ }^{\mathrm{cal}}(\mathrm{SS})=-5.8 \times 10^{3} \mathrm{~cm}^{-1}$, as compared with the experimental values, $\Delta \mathrm{E}_{1}{ }^{\exp }(\mathrm{SS})=-8.5 \times 10^{3} \mathrm{~cm}^{-1}$ and $\Delta \mathrm{E}_{2}{ }^{\exp }(\mathrm{SS})=-10.3 \times 10^{3} \mathrm{~cm}^{-1}$. Mostly due to the good ASEC description of the hydrogen bonds formed between the Prodan and the water molecules. The Stokes shifts of the high energy calculated here $\left(\Delta \mathrm{E}_{1}{ }^{\text {cal }}(\mathrm{SS})=-2.2 \times 10^{3}\right.$ $\mathrm{cm}^{-1}$ in vacuum and $\Delta \mathrm{E}_{1}{ }^{\mathrm{cal}}(\mathrm{SS})=-5.1 \times 10^{3} \mathrm{~cm}^{-1}$ in water) are also in agreement with the results obtained by Baral et $a l .{ }^{72}$ for the high energy of Prodan in hexane, $\Delta \mathrm{E}_{1}{ }^{\text {cal }}(\mathrm{SS})=-3.3 \mathrm{x}$ $10^{3} \mathrm{~cm}^{-1}(-0.41 \mathrm{eV})$ and in water, $\Delta \mathrm{E}_{1}{ }^{\mathrm{cal}}(\mathrm{SS})=-9.5 \times 10^{3} \mathrm{~cm}^{-1}(-1.18 \mathrm{eV})$. Their results are in better agreement with the experimental values $\left(\Delta \mathrm{E}_{1}{ }^{\exp }(\mathrm{SS})=-3.6 \times 10^{3} \mathrm{~cm}^{-1}\right.$ in cyclohexane and $\Delta \mathrm{E}_{1}{ }^{\exp }(\mathrm{SS})=-8.5 \times 10^{3} \mathrm{~cm}^{-1}$ in water). We are using $\mathrm{MM}$ simulation with the Prodan with rigid geometry and Baral et al. ${ }^{72}$ used flexible Prodan geometry, considering the geometry relaxation due to the solvent interactions. Therefore, it shows that the solvent effect on the geometry relaxation solute is important to described Stokes shift, but the dual fluorescence can be understood only analysing two excited states: the $\pi-\pi^{*} \mathrm{~L}_{\mathrm{a}}$ $\mathrm{HOMO} \rightarrow$ LUMO $\mathrm{S}_{1}$ state (high emission energy) and the $n-\pi * \mathrm{HOMO}-3 \rightarrow$ LUMO $+1 \mathrm{~S}_{2}$ or $\mathrm{S}_{3}$ state (low emission energy).

Now, analyzing the dipole moment of the low emission energy state, $\mu_{\mathrm{wat}}\left(n-\pi^{*}\right)=2.7$ $D$, and the high emission energy state, $\mu_{\mathrm{wat}}\left(\pi-\pi^{*} \mathrm{~L}_{\mathrm{a}}\right)=17.2 \mathrm{D}$ in water and $\mu_{\mathrm{vac}}\left(\pi-\pi^{*} \mathrm{~L}_{\mathrm{b}}\right)=5.8$ $\mathrm{D}$ in vacuum, it is easy to understand why the high emission state becomes more favorable increasing the solvent polarity. This is a consequence of a much stronger dipole interactions between the solvent molecules and the Prodan at the $\pi-\pi^{*} \mathrm{~L}_{\mathrm{a}}$ excited state than at the $n-\pi^{*}$ excited state.

\section{Conclusions}

The absorption spectra of Prodan in water, acetonitrile, dichloromethane and cyclohexane are well described with different methods (INDO-CIS, TDDFT and CASPT2) and all agree that the first band is composed of three different electronic transitions, one dark 
$n-\pi^{*}$ and two bright $\pi-\pi^{*}$. It is shown that the two bright excited electronic states can be simultaneously populated in the absorption transitions of Prodan.

Multi-configurational calculations showed that the three excited states, $\pi-\pi^{*} \mathrm{~L}_{\mathrm{b}}, n-\pi^{*}$ and $\pi-\pi * L_{a}$ are very close to each other in energy at the Franck-Condon geometry and their equilibrium structures are planar in vacuum. No twist of the carbonyl or dimethylamine groups is observed and they are in the same plane as the naphthalene rings. Considering the solvent effects in the electronic structure of the solute and in the solvent relaxation around the solute, it was identified that these three excited states can change order depending on the solvent polarity and following the minimum path energy, internal conversions may occur. The HOMO $\rightarrow$ LUMO $\mathrm{S}_{1}$ state $\left(\pi-\pi^{*} \mathrm{~L}_{b}\right.$ in vacuum or $\pi-\pi * \mathrm{~L}_{\mathrm{a}}$ in water) relaxes to the equilibrium geometry causing the high emission energy $\left(\mathrm{E}_{1}\right)$. The HOMO-3 $\rightarrow$ LUMO $+1 n-\pi^{*}$ state $\left(S_{2}\right.$ in vacuum or $S_{3}$ in water) also relaxes to the equilibrium geometry causing the low emission energy $\left(\mathrm{E}_{2}\right)$. The $\pi-\pi * \mathrm{~L}_{\mathrm{a}}$ state has a very large dipole moment $(\sim 17 \mathrm{D})$ and the $n-\pi *$ state has a very small dipole moment ( $\sim 2 \mathrm{D})$. This information of larger dipole to the high emission energy $E_{1}$ is in agreement with the experimental data that shows a larger contribution of $\mathrm{E}_{1}$ to the emission band in water comparatively to cyclohexane. Additionally, the calculated Stokes shifts of the high and low emission energy of Prodan in vacuum and in water are in agreement with the experimental data for cyclohexane and water, respectively.

Hence, our combined experimental and theoretical studies point to a new explanation of the dual fluorescence of molecular probes. We conclude that the Prodan probe does not emit from a solvent-non-relaxed locally excited state and a relaxed twist or planar internal charge transfer excited state. Instead, it fluoresces from two different and independent excited states, first $\pi-\pi^{*}$ state at high energy and $n-\pi^{*}$ state at low energy, which can be populated when Prodan is excited at 340-360 nm. Finally, it should be mentioned that this work focuses on the Prodan molecule but, we contend that the dual fluorescence of the related Laurdan molecule could be understood with the same reasoning.

\section{Availability of Data}

The data that supports the findings of this study are available within the article and its supplementary information material. 


\section{Dedication}

This paper honors Prof. Yvone Primerano Mascarenhas, a pioneer in the field of biomolecular structures in Brazil. With an outstanding capacity for teaching and mentoring, she inspired many students to pursue careers in biophysical chemistry thus helping to create important groups.

\section{Acknowledgments}

This work has been partially supported by the following Brazilian agencies and projects: FAPESP (2017/11631-2); CAPES for the BioMol project 23038.004630/2014-35; the National Institute of Science and Technology of Complex Fluids (INCT-FCx) with the CNPq grant 141260/2017-3 and FAPESP grant 2014/50983-3. CCV-S acknowledges fellowship supports from FAPESP 06/55513-9 and 10/08365-0. YOG acknowledges fellowship supports from FAPESP 12/15161-7. SC, MTL and KC acknowledge financial supports from CNPq.

\section{References}

1. G. Weber and F. J. Farris, Synthesis and Spectral Properties of a Hydrophobic Fluorescent-Probe - 6-Propionyl-2-(Dimethylamino)Naphthalene, Biochemistry, 1979, 18, 3075-3078.

2. T. Parasassi, F. Conti and E. Gratton, Time-Resolved Fluorescence Emission-Spectra of Laurdan in Phospholipid-Vesicles by Multifrequency Phase and Modulation Fluorometry, Cell Mol Biol, 1986, 32, 103-108.

3. T. Parasassi, G. Destasio, G. Ravagnan, R. Rusch and E. Gratton, Quantitation of Lipid Phases in Phospholipid-Vesicles by the Generalized Polarization of Laurdan Fluorescence, Biophys. J., 1991, 60, 179-189.

4. F. Moyano, J. J. Silber and N. M. Correa, On the investigation of the bilayer functionalities of 1,2-di-oleoyl-sn-glycero-3-phosphatidylcholine (DOPC) large unilamellar vesicles using cationic hemicyanines as optical probes: A wavelength-selective fluorescence approach, $J$ Colloid Interf Sci, 2008, 317, 332-345.

5. L. A. Bagatolli, B. Maggio, F. Aguilar, C. P. Sotomayor and G. D. Fidelio, Laurdan properties in glycosphingolipid-phospholipid mixtures: A comparative fluorescence and calorimetric study, Biochim. Biophys. Acta-Biomembranes, 1997, 1325, 80-90.

6. D. Marsh, Reaction Fields in the Environment of Fluorescent Probes: Polarity Profiles in Membranes, Biophys. J., 2009, 96, 2549-2558.

7. C. C. De Vequi-Suplicy, C. R. Benatti and M. T. Lamy, Laurdan in fluid bilayers: Position and structural sensitivity, J Fluoresc, 2006, 16, 431-439.

8. J. Catalan, P. Perez, J. Laynez and F. G. Blanco, Analysis of the Solvent Effect on the Photophysics Properties of 6-Propionyl-2-(dimethylamino)naphthalene (PRODAN), J Fluoresc, 1991, 1, 215-223.

9. J. R. Lakowicz, Principles of Fluorescence Spectroscopy, Plenum Publishers, New York, Third Edit., 2006.

10. C. C. Vequi-Suplicy, K. Coutinho and M. T. Lamy, New Insights on the Fluorescent Emission Spectra of Prodan and Laurdan, J. Fluoresc., 2015, 25, 621-629.

11. A. D. Lúcio, C. C. Vequi-Suplicy, R. M. Fernandez, M. T. Lamy, A. D. Lucio, C. C. Vequi- 
Suplicy, R. M. Fernandez, M. T. Lamy, Laurdan Spectrum Decomposition as a Tool for the Analysis of Surface Bilayer Structure and Polarity: a Study with DMPG, Peptides and Cholesterol, J. Fluoresc., 2010, 20, 473-482.

12. J. R. Lakowicz and A. Balter, Differential-Wavelength Deconvolution of Time-Resolved Fluorescence Intensities - a New Method for the Analysis of Excited-State Processes, Biophys. Chem., 1982, 16, 223-240.

13. J. R. Lakowicz and A. Balter, Analysis of Excited-State Processes by Phase-Modulation Fluorescence Spectroscopy, Biophys. Chem., 1982, 16, 117-132.

14. B. A. Rowe, C. A. Roach, J. Lin, V. Asiago, O. Dmitrenko, S. L. Neal, O. Dmitrenko, S. L. Neal, O. Dmitrenko and S. L. Neal, Spectral Heterogeneity of PRODAN Fluorescence in Isotropic Solvents Revealed by Multivariate Photokinetic Analysis, J. Phys. Chem. A, 2008, 112, 1340213412.

15. M. Raguz and J. Brnjas-Kraljevic, Resolved Fluorescence Emission Spectra of PRODAN in Ethanol/Buffer Solvents, J. Chem. Inf. Model. 2005, 45, 1636-1640.

16. T. Parasassi, E. K. Krasnowska, L. Bagatolli and E. Gratton, LAURDAN and PRODAN as polarity-sensitive fluorescent membrane probes, J. Fluoresc., 1998, 8, 365-373.

17. A. Balter, W. Nowak, W. Pawelkiewicz, A. Kowalczyk, W. Pawełkiewicz and A. Kowalczyk, Some remarks on the interpretation of the spectral properties of prodan, Chem. Phys. Lett., 1988, 143, 565-570.

18. C. E. Bunker, T. L. Bowen and Y. P. Sun, A Photophysical Study of Solvatochromic Probe 6Propionyl-2-(N,N-Dimethylamino)Naphthalene (Prodan) in Solution, Photochem Photobiol, 1993, 58, 499-505.

19. B. Mennucci, M. Caricato, F. Ingrosso, C. Cappelli, R. Cammi, J. Tomasi, G. Scalmani and M. J. Frisch, How the environment controls absorption and fluorescence spectra of PRODAN: A quantum-mechanical study in homogeneous and heterogeneous media, $J$ Phys Chem B, 2008, 112, 414-423.

20. L. Cwiklik, A. J. A. Aquino, M. Vazdar, P. Jurkiewicz, J. Pittner, M. Hof and H. Lischka, Absorption and Fluorescence of PRODAN in Phospholipid Bilayers: A Combined Quantum Mechanics and Classical Molecular Dynamics Study, J. Phys. Chem. A, 2011, 115, 11428-11437.

21. A. M. Rollinson and H. G. Drickamer, High-Pressure Study of Luminescence from Intramolecular Ct Compounds, J. Chem. Phys., 1980, 73, 5981-5996.

22. W. Nowak, P. Adamczak, A. Balter and A. Sygula, On the Possibility of Fluorescence from Twisted Intramolecular Charge-Transfer States of 2-Dimethylamino-6-Acylnaphthalenes - a Quantum-Chemical Study, Theochem-Journal Mol. Struct., 1986, 139, 13-23.

23. R. Adhikary, C. A. Barnes and J. W. Petrich, Solvation Dynamics of the Fluorescent Probe PRODAN in Heterogeneous Environments: Contributions from the Locally Excited and ChargeTransferred States, J Phys Chem B, 2009, 113, 11999-12004.

24. Y. P. Morozova, O. M. Zharkova, T. Y. Balakina and V. Y. Artyukhov, Effect of proton-donor solvent and structural flexibility of prodan and laurdan molecules on their spectral-luminescent properties, J. Appl. Spectrosc., 2009, 76, 312-318.

25. R. K. Everett, A. A. Nguyen, C. J. Abelt, H. A. A. Nguyen, C. J. Abelt, A. A. Nguyen and C. J. Abelt, Does PRODAN Possess an O-TICT Excited State? Synthesis and Properties of Two Constrained Derivatives, J. Phys. Chem. A, 2010, 114, 4946-4950.

26. B. N. Davis and C. J. Abelt, Synthesis and photophysical properties of models for twisted PRODAN and dimethylaminonaphthonitrile, J. Phys. Chem. A, 2005, 109, 1295-1298.

27. V. I. Tomin, Nonradiative energy transfer in a concentrated solution of prodan, Opt. Spectrosc., 2006, 101, 563-567.

28. V. I. Tomin and K. Hubisz, Inhomogeneous spectral broadening and the decay kinetics of the luminescence spectra of prodan, Opt. Spectrosc., 2006, 101, 98-104.

29. M. Novaira, M. A. Biasutti, J. J. Silber and N. M. Correa, New insights on the photophysical behavior of PRODAN in anionic and cationic reverse micelles: From which state or states does it emit?, J. Phys. Chem. B, 2007, 111, 748-759.

30. P. Ilich and F. G. Prendergast, Singlet adiabatic states of solvated PRODAN: A semiempirical molecular orbital study, J. Phys. Chem., 1989, 93, 4441-4447.

31. A. B. J. Parusel, F. W. Schneider and G. Kohler, An ab initio study on excited and ground state 
properties of the organic fluorescence probe PRODAN, J Mol Struc-Theochem, 1997, 398, 341346.

32. M. Viard, J. Gallay, M. Vincent, O. Meyer, B. Robert and M. M. Paternostre, Laurdan solvatochromism: Solvent dielectric relaxation and intramolecular excited-state reaction, Biophys. $J ., 1997, \mathbf{7 3}, 2221-2234$.

33. A. Parusel, Semiempirical studies of solvent effects on the intramolecular charge transfer of the fluorescence probe PRODAN, J. Chem. Soc. Trans., 1998, 94, 2923-2927.

34. A. B. J. J. Parusel, R. Schamschule, G. Köhler, G. Ko, A. B. J. J. Parusel, R. Schamschule and G. Kohler, Nonlinear optics. A semiempirical study of organic chromophores, J. Mol. Struct. THEOCHEM, 2001, 544, 253-261.

35. K. A. Kozyra, J. R. Heldt, J. Heldt, M. Engelke and H. A. Diehl, Concentration and temperature dependence of Laurdan fluorescence in glycerol, Z Naturforsch. A, 2003, 58, 581-588.

36. B. C. Lobo and C. J. Abelt, Does PRODAN possess a planar or twisted charge-transfer excited state? Photophysical properties of two PRODAN derivatives, J. Phys. Chem. A, 2003, 107, 10938-10943.

37. M. Novaira, F. Moyano, M. A. Biasutti, J. J. Silber and N. M. Correa, An example of how to use AOT reverse micelle interfaces to control a photoinduced intramolecular charge-transfer process, Langmuir, 2008, 24, 4637-4646.

38. A. Samanta and R. W. Fessenden, Excited state dipole moment of PRODAN as determined from transient dielectric loss measurements, J. Phys. Chem. A, 2000, 104, 8972-8975.

39. K. Coutinho, S. Canuto and M. C. Zerner, A Monte Carlo-quantum mechanics study of the solvatochromic shifts of the lowest transition of benzene, J. Chem. Phys., 2000, 112, 9874-9880.

40. W. R. Rocha, K. Coutinho W. B. De Almeida and S. Canuto, An Efficient Quantum Mechanical/Molecular Mechanics Monte Carlo Simulation of Liquid Water, Chem. Phys. Lett. $2001, \mathbf{3 3 5}, 127$.

41. W. R. Rocha, V. M. Martins, K. Coutinho and S. Canuto, Solvent effects on the electronic absorption spectrum of formamide studied by a sequential Monte Carlo/quantum mechanical approach, Theor. Chem. Acc., 2002, 108, 31-37.

42. S. Miertus, E. Scrocco and J. Tomasi, Electrostatic Interaction of a Solute with a Continuum - a Direct Utilization of Abinitio Molecular Potentials for the Prevision of Solvent Effects, Chem. Phys., 1981, 55, 117-129.

43. C. C. Vequi-Suplicy, K. Coutinho and M. T. Lamy, Electric dipole moments of the fluorescent probes Prodan and Laurdan: experimental and theoretical evaluations, Biophys. Rev., 2014, 6, 6374.

44. H. C. Georg, K. Coutinho and S. Canuto, Converged electronic polarization of acetone in liquid water and the role in the n-pi* transition, Chem. Phys. Lett., 2006, 429, 119-123.

45. R. C. Barreto, K. Coutinho, H. C. Georg and S. Canuto, Combined Monte Carlo and quantum mechanics study of the solvatochromism of phenol in water. The origin of the blue shift of the lowest pi-pi* transition., Phys. Chem. Chem. Phys., 2009, 11, 1388-1396.

46. V. Manzoni, M. L. Lyra, R. M. Gester, K. Coutinho and S. Canuto, Study of the optical and magnetic properties of pyrimidine in water combining PCM and QM/MM methodologies, Phys. Chem. Chem. Phys, 2010, 12, 14023.

47. K. Coutinho, H. C. Georg, T. L. Fonseca, V. Ludwig and S. Canuto, An efficient statistically converged average configuration for solvent effects, Chem. Phys. Lett., 2007, 437, 148-152.

48. C. M. Breneman and K. B. Wiberg, Determining Atom-Centered Monopoles from Molecular Electrostatic Potentials - the Need for High Sampling Density in Formamide ConformationalAnalysis, J Comp Chem, 1990, 11, 361-373.

49. N. Ferre and J. G. Angyan, Approximate electrostatic interaction operator for QM/MM calculations, Chem. Phys. Lett., 2002, 356, 331-339.

50. Y. Orozco-Gonzalez, C. Bistafa and S. Canuto, Solvent Effect on the Stokes Shift and on the Nonfluorescent Decay of the Daidzein Molecular System, J. Phys. Chem. A, 2013, 117, 44044411.

51. E. Runge and E. K. U. Gross, Density-Functional Theory for Time-Dependent Systems, Phys. Rev. Lett., 1984, 52, 997-1000.

52. J. Ridley and M. Zerner, Intermediate Neglect of Differential Overlap Technique for 
Spectroscopy - Pyrrole and Azines, Theor. Chim. Acta, 1973, 32, 111-134.

53. J. Tomasi, Thirty years of continuum solvation chemistry: a review, and prospects for the near future, Theor Chem Acc, 2004, 112, 184-203.

54. J. Tomasi, B. Mennucci and R. Cammi, Quantum mechanical continuum solvation models, Chem. Rev., 2005, 105, 2999-3093.

55. K. Andersson, P. A. Malmqvist and B. O. Roos, 2nd-Order Perturbation-Theory with a Complete Active Space Self-Consistent Field Reference Function, J. Chem. Phys., 1992, 96, 1218-1226.

56. F. Aquilante, L. De Vico, N. Ferre, G. Ghigo, P. A. Malmqvist, P. Neogrady, T. B. Pedersen, M. Pitonak, M. Reiher, B. O. Roos, L. Serrano-Andres, M. Urban, V. Veryazov and R. Lindh, Software News and Update MOLCAS 7: The Next Generation, J. Comput. Chem., 2010, 31, 224 247.

57. C. Centre, Theoretica Chimica Acta Density matrix averaged atomic natural orbital (ANO) basis sets for correlated molecular wave functions, Theor. Chim. Acta, 1990, 77, 291-306.

58. P.-O. O. Widmark, B. Joakim, Persson, B. O. Roos, B. J. Persson and B. O. Roos, Density matrix averaged atomic natural orbital (ANO) basis sets for correlated molecular wave functions, Theor. Chim. Acta, 1991, 79, 419-432.

59. M. C. Zerner, ZINDO/UF: A semi-empirical program package, 2000, University of Florida, Gainesville/USA.

60. M. J. Frisch, G. W. Trucks, H. B. Schlegel, G. E. Scuseria, M. A. Robb, J. R. Cheeseman, J. A. Montgomery, T. V. Jr., K. N. Kudin, J. C. Burant, J. M. Millam, J. A. Pople, et al., 2004, GAUSSIAN 03.

61. G. Karlstrom, R. Lindh, P. A. Malmqvist, B. O. Roos, U. Ryde, V. Veryazov, P. O. Widmark, M. Cossi, B. Schimmelpfennig, P. Neogrady and L. Seijo, MOLCAS: a program package for computational chemistry, Comput. Mater. Sci., 2003, 28, 222-239.

62. M. P. Allen and D. J. Tildesley, Computer Simulation of Liquids, Oxford University Press, Oxford, 1989.

63. W. L. Jorgensen, D. S. Maxwell and J. TiradoRives, Development and testing of the OPLS allatom force field on conformational energetics and properties of organic liquids, J. Am. Chem. Soc., 1996, 118, 11225-11236.

64. H. J. C. C. Berendsen, J. R. Grigera and T. P. Straatsma, The Missing Term in Effective Pair Potentials, J. Phys. Chem., 1987, 91, 6269-6271.

65. H. J. Bohm, I. R. Mcdonald and P. A. Madden, An Effective Pair Potential for Liquid Acetonitrile, Mol. Phys., 1983, 49, 347-360.

66. W. C. Swope, H. C. Andersen, P. H. Berens and K. R. Wilson, A computer simulation method for the calculation of equilibrium constants for the formation of physical clusters of molecules: Application to small water clusters, J. Chem. Phys., 1982, 76, 637-649.

67. H. J. C. Berendsen, J. P. M. Postma, W. F. Vangunsteren, A. Dinola and J. R. Haak, MolecularDynamics with Coupling to an External Bath, J. Chem. Phys., 1984, 81, 3684-3690.

68. K. Coutinho and S. Canuto, 2009, DICE: A Monte Carlo program for molecular simulation, University of São Paulo, SP, Brazil.

69. H. M. Cezar, S. Canuto and K. Coutinho, DICE: A Monte Carlo code for molecular simulation including Configurational Bias Monte Carlo method, J. Chem. Inf. Model. 2020, 60, 3472-3488.

70. J. W. Ponder and F. M. Richards, An Efficient Newton-Like Method for Molecular Mechanics Energy Minimization of Large Molecules, J Comp Chem, 1987, 8, 1016-1024.

71. C. E. Kundrot, J. W. Ponder and F. M. Richards, Algorithms for Calculating Excluded Volume and Its Derivatives as a Function of Molecular-Conformation and Their Use in Energy Minimization, J Comp Chem, 1991, 12, 402-409.

72. S. Baral, M. Phillips, H. Yan, J. Avenso, L. Gundlach, B. Baumeier and E. Lyman, Ultrafast Formation of the Charge Transfer State of Prodan Reveals Unique Aspect of the Chromophore Environment, J. Phys. Chem. B 2020, 124, 2643-2651.

73. H. C. Georg, K. Coutinho, and S. Canuto, Solvent effects on the UV-visible absorption spectrum of benzophenone in water: A combined Monte Carlo quantum mechanics study including solute polarization, J. Chem. Phys. 2007, 1261, 034507.

74. S. Canuto, K. Coutinho and D. Trzesniak, New developments in Monte Carlo/quantum mechanics methodology. The solvatochromism of beta-carotene in different solvents, Adv Quantum Chem, 
2002, 41, 161-183.

75. Y. Huang, X. Y. Li, K. X. Fu and Q. Zhu, New formulation for non-equilibrium solvation: Spectral shifts and cavity radii of 6-propanoyl-2(N,N-dimethylamino) naphthalene and 4-(N,Ndimethylamino) benzonitrile, J. Theor. Comput. Chem., 2006, 5, 355-374. 